\title{
Configuration Synthesis and Performance Analysis of 9-Speed Automatic Transmissions
}

\author{
Huafeng Ding ${ }^{1,2,3^{*}} \mathbb{D}$, Changwang Cai ${ }^{1,2}$, Ziming Chen ${ }^{1,2}$, Tao Ke $^{3}$ and Bowen Mao ${ }^{1}$
}

\begin{abstract}
Current research of automatic transmission (AT) mainly focuses on the improvement of driving performance, and configuration innovation is one of the main research directions. However, finding new configurations of ATs is one of the main limitations of configuration innovation. In the present study, epicyclic gear trains (EGTs) are applied to investigate mechanisms of 9-speed ATs. Then four kinematic configurations are proposed for automatic transitions. In order to evaluate the performance of proposed mechanisms, the lever analogy method is applied to conduct kinematic and mechanical analyses. The power flow analysis is conducted, and then transmission efficiencies are calculated based on the torque method. The comparative analysis between the proposed and existing mechanisms is carried out where obtained results show that proposed mechanisms have reasonable performance and can be used in ATs. The prototype of an AT is manufactured and the speed test is conducted, which proves the accuracy of analysis and the feasibility of proposed mechanisms.
\end{abstract}

Keywords: Automatic transmission, Epicyclic gear train, Lever analogy, Gear ratio, Torque method

\section{Introduction}

In the automotive industry, vehicles equipped with automatic transmissions (ATs) have significant advantages, including simple operation, smooth gear shift and long service life. Moreover, the corresponding driving performance and ride comfort are significantly improved compared to cars with manual transmissions. The first cars equipped with the Hydra-Matic ATs were developed and put into market by General Motors in 1940s. Since then, cars equipped with automatic transmissions have attracted the vast number of consumers, and quickly occupied the automotive market. This is especially more pronounced in United States, European countries and Japan [1]. Along with the continuous development of the automotive industry, the innovation and development of ATs is of great significance.

In the past few decades, many innovations have been proposed in the area of automatic transmissions.

\footnotetext{
*Correspondence: dhf@ysu.edu.cn

1 School of Mechanical Engineering, Yanshan University,

Qinhuangdao 066004, China

Full list of author information is available at the end of the article
}

Nowadays, the epicyclic-type ATs are the most widely applied ATs, which originates from its remarkable superiorities, including compact structure, large gear ratios, strong bearing capacity and long-life operation. The first step in the conceptual design phase of the AT is the selection of kinematic configurations to provide desired gear ratios [2]. An epicyclic-type AT mechanism typically consists of the hydraulic transmission and the mechanical transmission parts. The hydraulic transmission part mainly includes a torque converter, while the mechanical transmission part includes an epicyclic gear train (EGT) and a set of shifting elements such as clutches and brakes. In order to obtain kinematic configurations of ATs, scholars have proposed synthesis methods for EGTs. Johnson and Towfigh [3] utilized the synthetic approach of linkage type kinematic chains and proposed a synthesis method for EGTs. Then they synthesized gear mechanisms with one degree of freedom (DOF) with up to 8 links. Moreover, Buchsbaum and Freudenstein [4] and Freudenstein [5] applied the graph theory into the synthesis process of geared kinematic chains (GKCs), and obtained epicyclic gear chains with up to 5 links. Ravisankar and Mruthyunjaya [6] applied the graph theory and matrix to 
synthesize GKCs and obtained all 1-DOF EGTs with up to 4 fundamental loops. Furthermore, Tsai et al. [7-10] proposed an isomorphism detection method by computing characteristic polynomial of EGTs, the genetic graph approach to synthesize non-isomorphic EGTs with up to 6 links and non-fractionated 2-DOF EGTs with up to 7 links, and the canonical graph representation of GKCs to solve the pseudo isomorphic problem. Kim and Kwak [11] employed a recursive method and synthesized EGTs with up to 7 links. Hsu and Lam [12] presented a new graph representation for planetary gear trains (PGTs). Then Hsu [13] proposed a synthesis method for GKCs based on the new graph representation. Based on certain functional constraints, Castillo [14] proposed a synthesis method and then he applied the proposed method to synthesize 1-DOF PGTs with up to 9 links. Moreover, Ngo and Yan [15] generated 48 configurations and synthesized series-parallel hybrid transmissions for vehicles in the public transportation. Rajasri [16] proposed an approach in accordance with the hamming number and generated EGTs with up to 7 links. Kamesh [17] applied the vertex incidence polynomial and proposed a synthesis method. Then he reported all 1-DOF non-isomorphic EGTs with up to 6 links. Shanmukhasundaram et al. [18] applied the concept of kinematic unit in the representation method of rotation and displacement graphs to synthesize EGTs with up to 7 links. Moreover, scholars [19-21] focused on the configurations synthesis of ATs, which is normally used for hybrid electric vehicles. It should be indicated that these devices are expected to play an important role in the energy and environmental conservations in the near future. Reviewing the literature indicates that diverse EGTs have been proposed so far, which provide numerous kinematic configurations for innovative ATs. Nowadays, 6-, 7- and 8-speed ATs are mainly products in the automotive industry, while some 9-speed ATs are under test and further development. Along with the development of the AT technology, EGTs with more links are highly demanded to design ATs with more gears.

On the other hand, it is of significant importance to evaluate the feasibility and performance of AT mechanisms from different aspects, including the kinematic and dynamic analysis. To this end, different methods such as the relative velocity method [22], the lever analogy method [23] and the topology-based method [24] have been proposed. These methods can predict the speed, speed ratio and force condition of the candidate AT as an appropriate evaluation index. Reviewing the literature indicates that the lever analogy method is widely applied to analyze speeds and force condition of EGTs. Then the power flow and power loss are analyzed to obtain another evaluation index, called the transmission efficiency [2,
25-28]. In fact, the torque method [29], applying the concept of the ratio sensitivity [30,31], is a simple and universal scheme, which can be applied to all structures.

Ding et al. [32-36] presented unified topological representation models for planar kinematic chains. Then they synthesized EGTs with up to 9 links and established a topological graph database for EGTs. In the present study, it is intended to propose a synthesis method based on the topological graph database. Then the proposed method was applied to investigate the kinematic configurations of different ATs, covering existing designs and new designs. In order to illustrate the synthesis process, 9-speed ATs were synthesized and four mechanisms were proposed. Then, the lever analogy method was applied to conduct the kinematic and mechanic analyses of the proposed configurations, as well as the power flow analysis. Moreover, the transmission efficiencies were calculated through the torque method. In order to evaluate the feasibility and performance of the proposed mechanisms, comparative analysis was carried out. Finally, the prototype of one of mechanisms with the best performance was manufactured and the speed test experiment was conducted.

\section{Configuration Synthesis of 9-speed AT Mechanisms}

Studies show that as the number of gear ratios in the ATs increases, the fuel consumptions rate reduces and ride comfort improves [37]. Nowadays, ATs with 6-8 gears are mainstream products in the automotive industry. Meanwhile, 9-speed ATs are under test and development. Figure 1 illustrates configurations of different 9-speed automatic transmissions. Among the presented schemes, the ZF 9HP consists of four deceleration gears, one direct gear, four over-speed gears and one reverse gear, while the Benz 9G-Tronic consists of five deceleration gears, one direct gear, three over-speed gears and one reverse gear. Moreover, the GM 9T50E consists of six deceleration gears, one direct gear, two over-speed gears and one reverse gear.

Configuration of EGTs, as the key component of AT mechanism, is of significant importance for the designing ATs. Based on the unified topological presentation models of GKCs proposed by Ding [32], EGTs used in ATs can be presented by double bicolor graphs (DBGs). For the EGTs with one main shaft, the number of vertices of DBGs is one more than the number of links of corresponding EGTs. Moreover, the mobility of DBGs is one more than that of corresponding EGTs. For example, the structure diagrams of ATs shown in Figure 1 are obtained from Refs. [38-40], and the corresponding EGTs are converted into DBGs, where the results are presented 


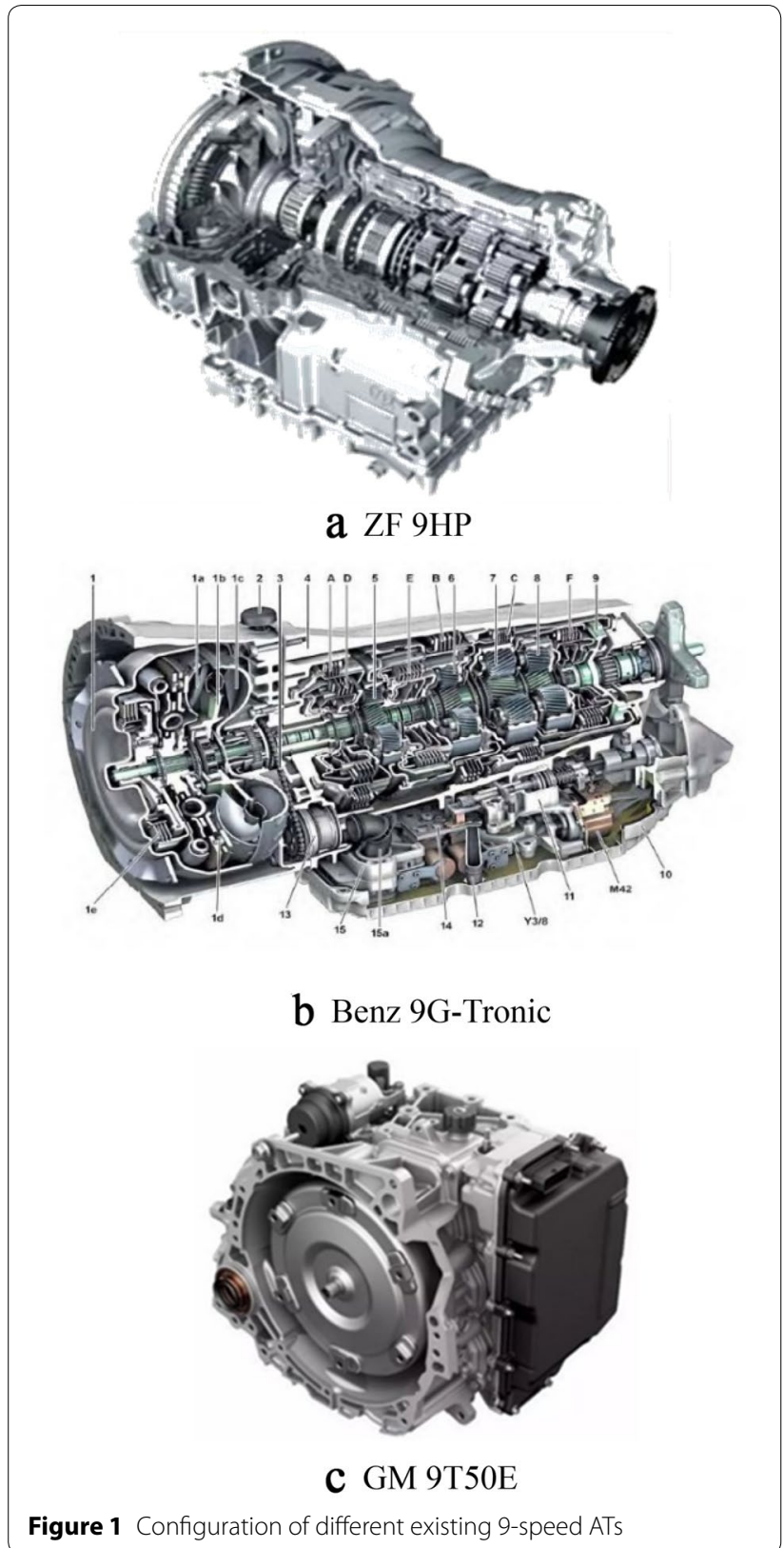

in Figure 2. It is observed that the EGT used in ZF 9HP is 2-DOF and 11-link, while the corresponding DBG is 3 -DOF and has 12 vertices.

Ding et al. [33] established a topological graph database for EGTs. They synthesized EGTs with up to 11 links and presented them through corresponding DBGs in the database. EGTs for ZF 9HP, Benz 9G-Tronic and GM 9T50E schemes have 11 links, 12 links and 10 links, respectively. Considering the number of vertices and the mobility of the corresponding DBGs, EGTs used in ZF 9HP and GM 9T50E can be obtained from

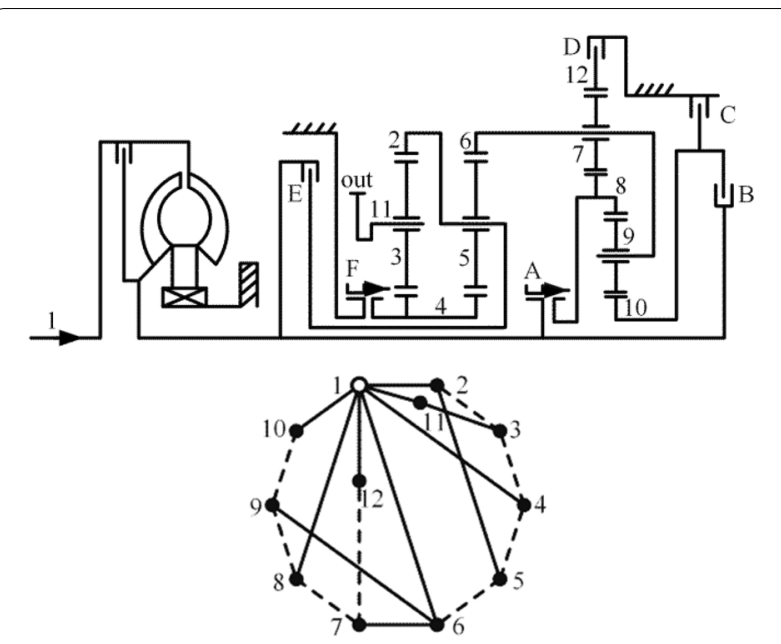

a ZF 9HP

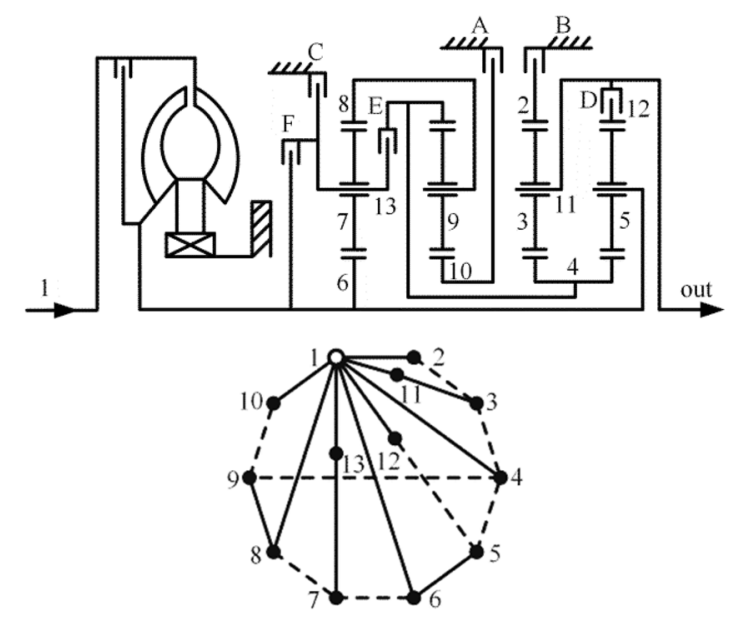

b Benz 9G-Tronic
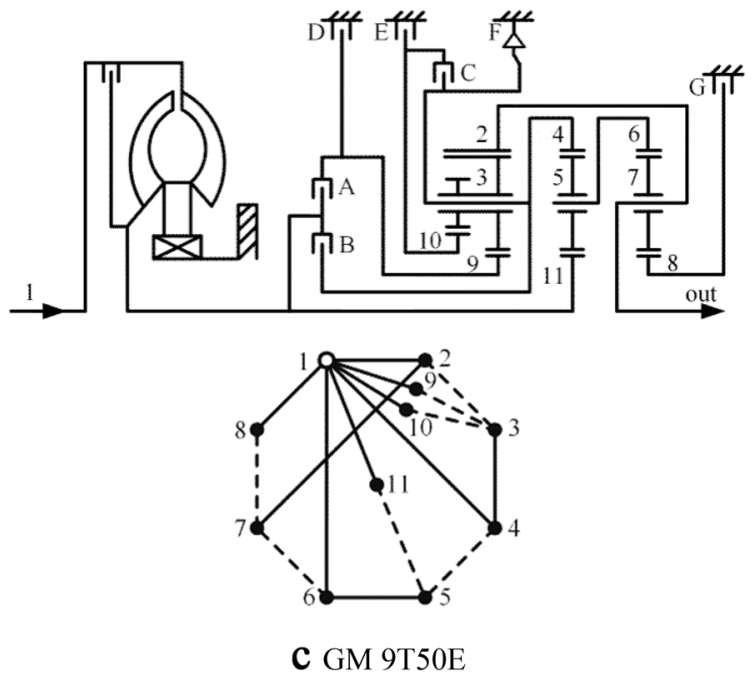

Figure 2 Structure diagrams of different ATs and the corresponding DBGs 


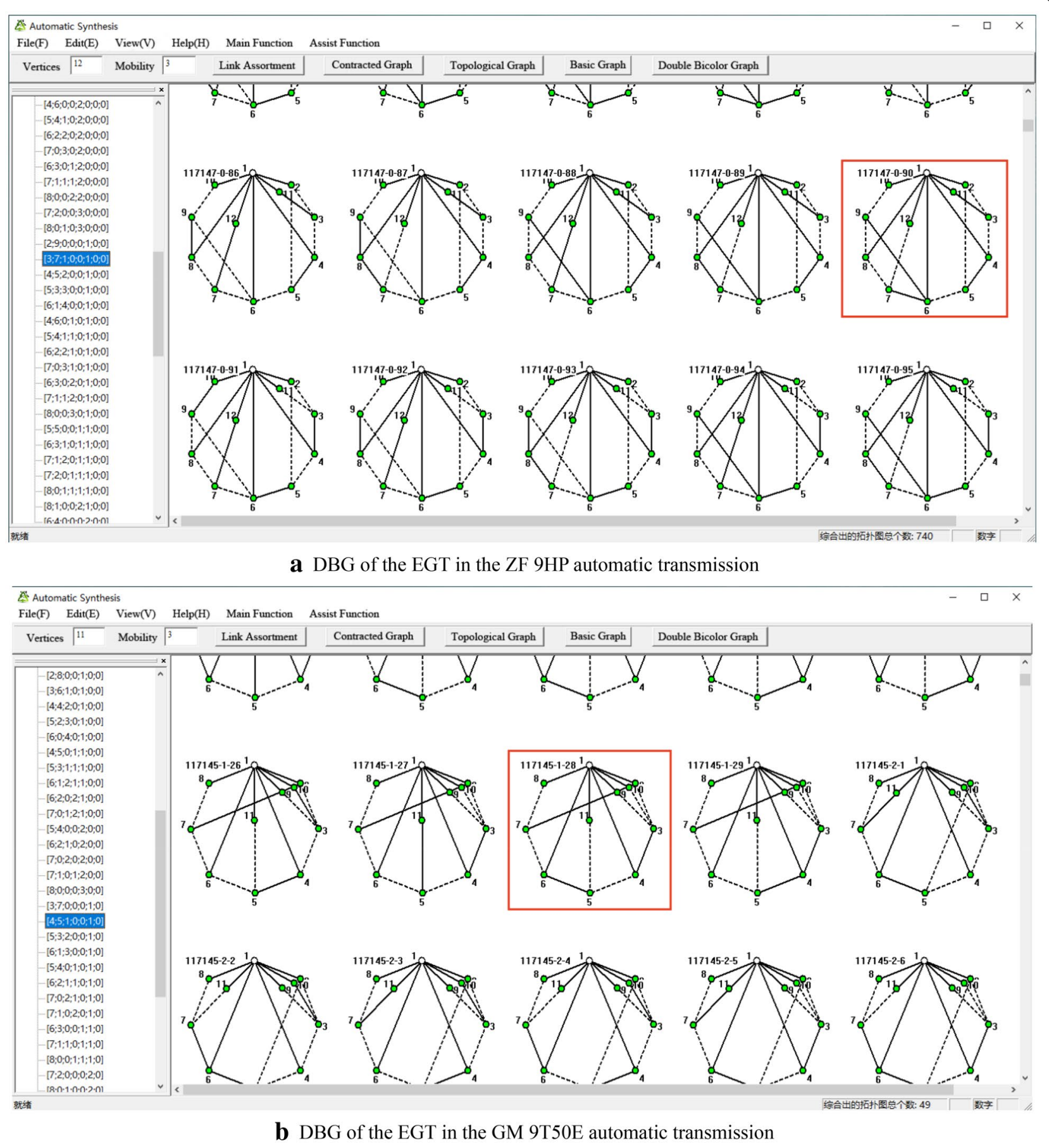

Figure 3 DBGs of EGTs in different automatic transmissions

the topological graph database as shown in Figure 3. It should be indicated that there are some DBGs similar to the existing EGTs in topological features, which may be used to obtain AT mechanisms having similar kinematic structures with the existing AT products.
It should be indicated that AT mechanisms with certain requirements can be obtained from the scratch through the topological graph database. For EGTs used in AT mechanisms, only coaxial links, namely central gears (sun and ring gears) and carriers, can be used as the input, output, or fixed components to obtain the 
desired gear ratios [41]. For a 1-DOF AT mechanism with $n_{c}$ coaxial links, the number of all possible gear ratios, including the direct drive, is presented by $m$. When the output is specified, $m$ can be calculated through the following expression:

$$
m=\left(n_{c}-1\right)\left(n_{c}-2\right)+1 .
$$

The mobility of AT mechanism is defined as the number of components that can rotate freely when all of shifting elements are in the separate state. In an AT mechanism, a shifting element is called clutch when applied to connect two different components, or called brake when applied to fix one component to the housing. This shifting element reduces the mobility of AT mechanism from $F$ to $(F-1)$ and the number of coaxial links from $n_{c}$ to $\left(n_{c}-1\right)$, respectively. Therefore, for an AT mechanism with $F$ degrees of freedom, $(F-1)$ shifting elements are required to reduce the mobility of the mechanism to 1 . Meanwhile, the entire movement of the AT mechanism is determined for a certain input speed so that the desired gear ratio can be obtained [42].

In order to obtain a 9-speed AT mechanism, the number of desired gear ratios, including the reverse gear, is 10 . Then, the number of coaxial links $n_{c}$ in a 1-DOF 9-speed AT mechanism should satisfy the following inequalities:

$$
\begin{aligned}
& \left(n_{c}-1\right)\left(n_{c}-2\right)+1 \geq 10, \\
& n_{c} \geq 5 .
\end{aligned}
$$

In order to obtain 9-speed AT mechanisms with $F$ degrees of freedom, the number of coaxial links should be more than $(4+F)$. It should be indicated that based on the abovementioned definition for the AT mechanism mobility and the Chebychev-Grübler-Kutzbach criterion for planar mechanisms [43], the mobility of EGTs used in $F$-DOF AT mechanisms is $(F-1)$. In the present study, EGTs satisfying the relationship between their mobility and the number of coaxial links are synthesized and shifting elements are added to obtain the corresponding configurations for designing 9-speed AT mechanisms.

For convenience of control and smooth shifting operation, two shifting elements are simultaneously applied in the mechanism. Moreover, the "clutch-clutch" shifting principle is considered. In this scheme, one shifting element maintains the contact unchanged, while the other shifting element disconnects. At the same time, one of the remaining shifting elements is applied to be working state. In the present study, 9-speed AT mechanisms with 3-DOF are considered to illustrate the synthesis process. To this end, the number of coaxial links should exceed 7.

At the first step, the mobility and the number of vertices of the corresponding DBGs are set in accordance with the graph database. Then the possible link assortments are obtained. For example, when 2-DOF EGTs with 11 links are considered, the corresponding 3-DOF DBGs have 12 vertices. In this case, the number of vertex and mobility in the topological graph database are set to 12 and 3, respectively. And 103 possible link assortments are obtained. Figure 4 illustrates the obtained link assortments.

At the second step, the maximum value of degree of vertices of DBGs should be detected in each link assortment. When the detected value is equal to or more than $(4+F)$, DBGs in the link assortment are synthesized. Figure 5 illustrates some of obtained DBGs for 11-link 2-DOF EGTs with 7 coaxial links.

Considering the characteristics of topology graphs and practical applications of EGTs, DBGs with the following conditions should be eliminated.

(1) DBGs with rigid sub-chains;

(2) Non-planar DBGs;

(3) DBGs with vertices connecting to a hollow vertex through dash lines;

(4) DBGs with vertices, which are only adjacent to dash lines;

(5) When the number of dash lines adjacent to a vertex representing a planet gear is less than two.

DBGs satisfying the foregoing constraints are eliminated. Then, four DBGs are selected as examples as shown in Figure 6.

At the third step, in order to conduct the performance analysis and the arrangement of shifting elements, it is necessary to draw function diagrams of EGTs corresponding to the DBGs. In the present study, the Buchsbaum-Freudenstein method [4] is applied for converting DBGs to function diagrams of EGTs. Then, the carriers, planet gears, sun gears and ring gears are determined in accordance with the correspondences between DBGs and EGTs. The DBGs shown in Figure 6 are converted to the function diagrams as shown in Figure 7.

Figure 7 indicates that EGTs (1) and (2) are structurally similar. Furthermore, EGTs (3) and (4) are structurally similar. It should be indicated that structurally similar EGTs for any existing AT can be found from the database.

Finally, configurations of 9-speed AT mechanisms can be obtained by adding shifting elements through the lever analogy method [23]. This can be carried out as following:

(1) Transform the function diagrams of EGTs into equivalent lever diagrams through the lever analogy method. 
Figure 4 Link assortments of 12-vertex 3-DOF DBGs obtained from the topological graph database

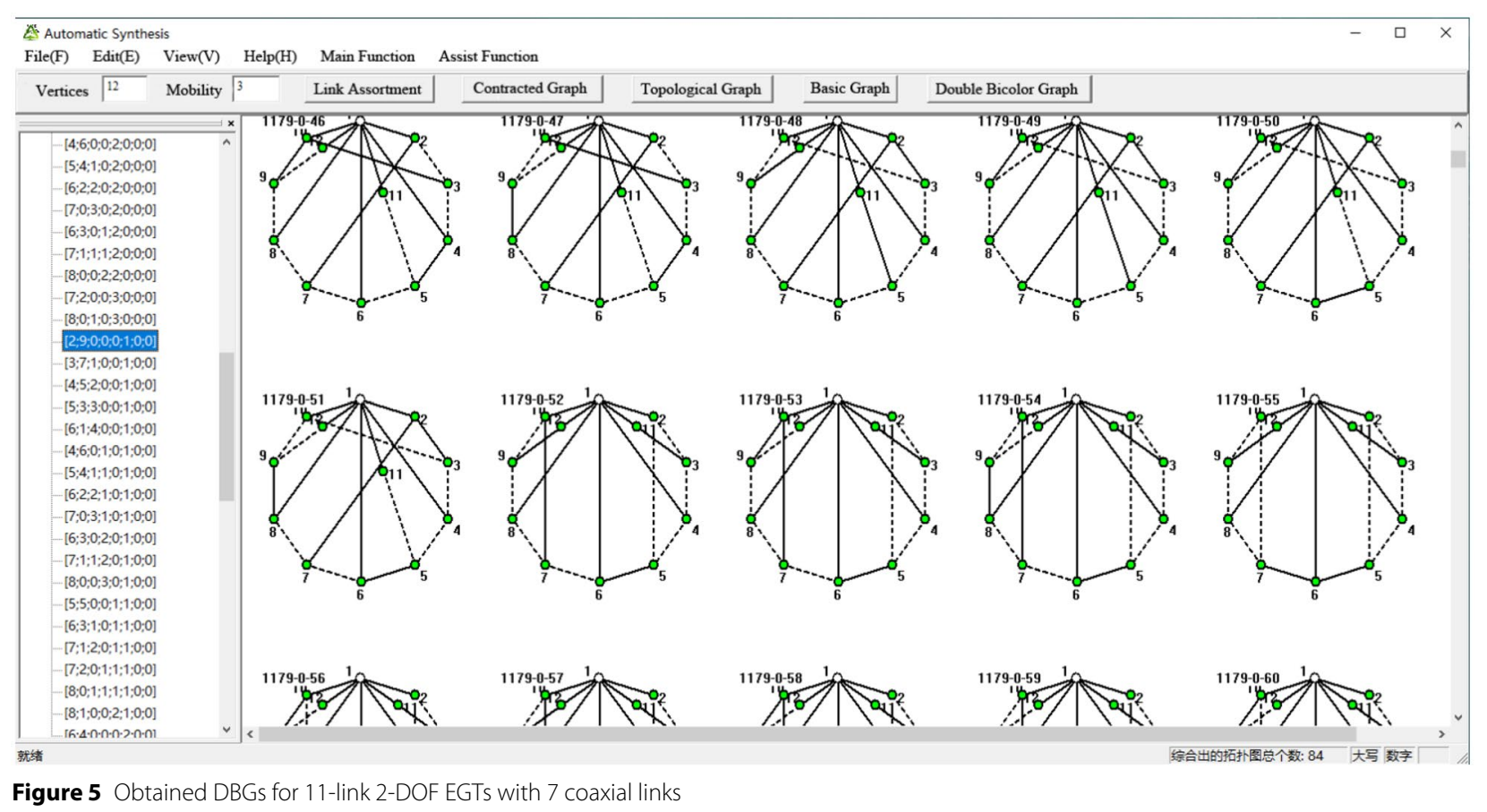

(2) Determine the output member, which is normally the carrier or the ring gear.

(3) Determine the input member, which is normally the sun gear or the carrier.
(4) Determine the position of clutches. Clutches have two operating modes: The first mode connects components of two different planetary gear sets (PGSs), namely the simple planetary gear train 


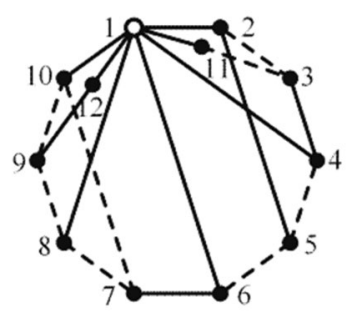

(1)

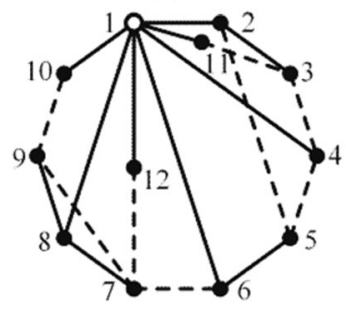

(3)

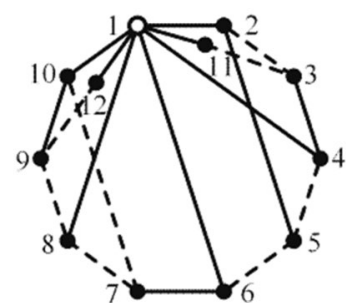

(2)

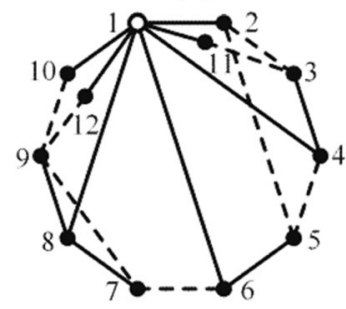

(4)

Figure 6 Four DBGs selected from link assortments $[2 ; 9 ; 0 ; 0 ; 0 ; 1]$ and $[3 ; 7 ; 1 ; 0 ; 0 ; 1]$

(1)

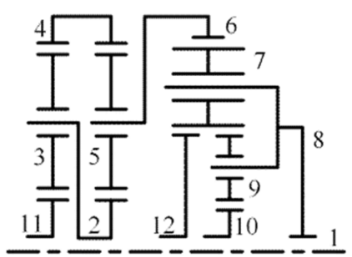

(3)

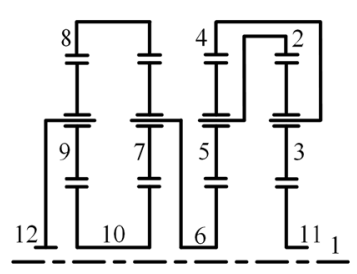

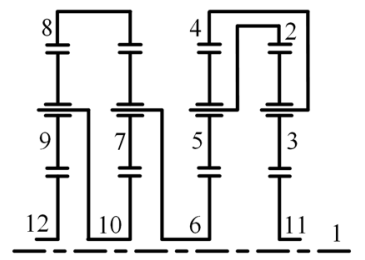

(2)

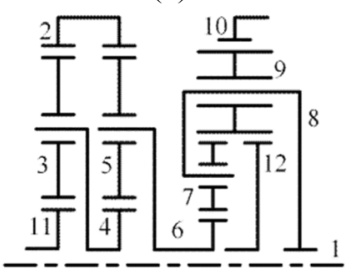

(4)
Figure 7 Function diagrams of four DBGs presented in Figure 6

defined by Lévai [22]. The second mode connects two different components of one PGS to form an atresia. The main purpose of the first mode is to reduce the mobility of EGTs, while the second mode is always employed to achieve the direct gear. When the second mode is applied, all components of the PGS rotate as a whole, called atresia.

(5) Determine the position of brakes. Brakes cannot be added on input member and output member.

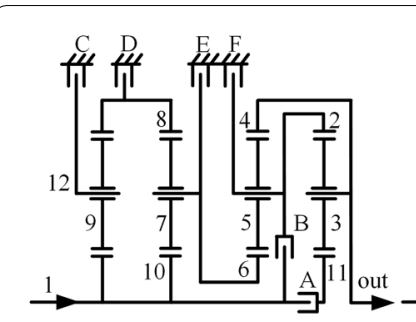

(1)

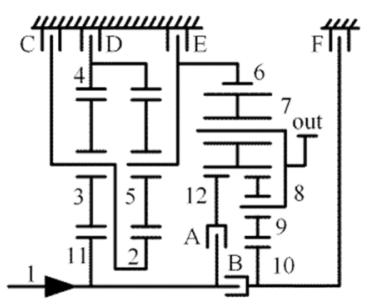

(3)

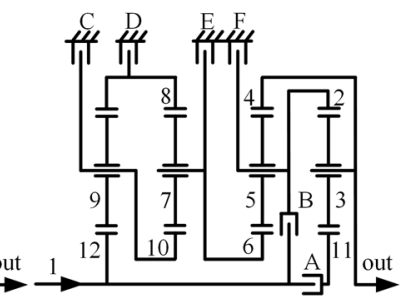

(2)

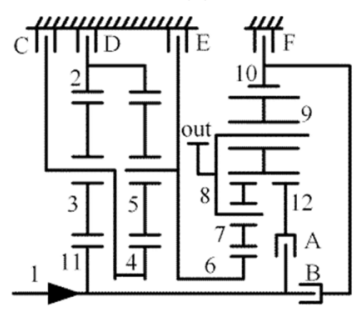

(4)
Figure 8 The structure diagrams of AT mechanisms converted from DBGs presented in Figure 6

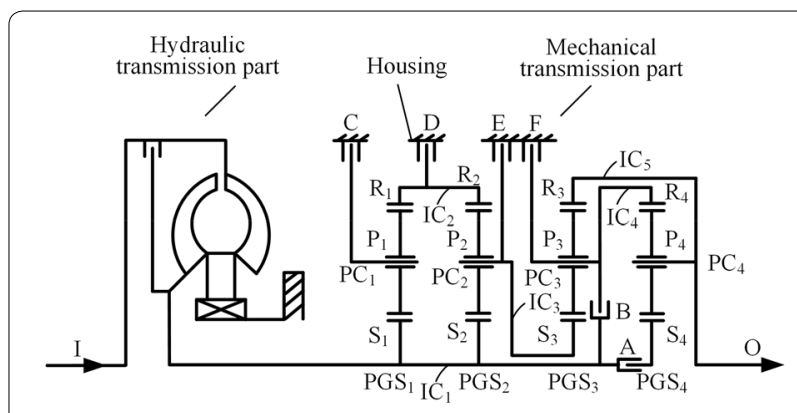

Figure 9 Structural diagram of the AT mechanism (1)

In the present study, foregoing steps are applied for the conversion. Presented DBGs in Figure 6 are converted into AT mechanisms and the corresponding structure diagrams are shown in Figure 8.

Reviewing the literature indicates that these schemes have not been proposed before. Therefore, they have been or going to be patented [44-47].

\section{Performance Analysis of Proposed AT Mechanisms}

Studies show that the variation range of gear ratios, interval of gear ratios and the transmission efficiency are important indexes to evaluate the performance of AT mechanisms. On the other hand, the mechanical performance of AT mechanisms has an important influence on the performance and service life of the system. In this section, it is intended to analyze the performance of the 
proposed AT mechanisms shown in Figure 8. To this end, the AT mechanism (1) is taken as the example to illustrate the analyzing process. Figure 9 illustrates the structure of the AT mechanism (1) in detail.

Figure 9 indicates that the AT mechanism mainly consists of the hydraulic transmission and the mechanical transmission parts. It is observed that these two parts are installed in the housing. Moreover, the hydraulic transmission part mainly consists of a torque converter, while the mechanical transmission part consists of an EGT and six shifting elements. The EGT is made from four PGSs (called $\mathrm{PGS}_{1}-\mathrm{PGS}_{4}$ ) and five interconnecting components (called $\mathrm{IC}_{1}-\mathrm{IC}_{5}$ ). Furthermore, shifting elements include two clutches (i.e., A, B) and four brakes (i.e., C, D, E, F). Power from input shaft (I) transmits through hydraulic transmission part and mechanical transmission part to the output shaft $(\mathrm{O})$ so that the vehicle moves with the expected speed.

Figure 9 indicates that each PGS consists of four members, including the sun gear $(\mathrm{S})$, ring gear $(\mathrm{R})$, planet gear $(\mathrm{P})$ and the carrier (PC). These members are connected to each other through ICs and shifting elements. Each IC operates like a coupling and connects two members between two PGSs to form a component all the time. On the other hand, a shifting element connects or separates the components in different gears. Different gear ratios can be obtained through connecting or separating different shifting elements selectively. It should be indicated that the proposed AT mechanism has ten different gear ratios (including a reverse gear), so that it is categorized as a 9 -speed transmission.

\subsection{Kinematic Analysis}

The kinematic analysis mainly includes the calculation of the gear ratio and the rotational speeds of the moving components at each gear so that the range and interval of gear ratios can be obtained. The gear ratio of the AT mechanism refers to the ratio of the input shaft speed to the output shaft speed. The absolute value of the gear ratio indicates the size, while the corresponding sign indicates the correlation between the rotation direction of the input and the output shafts.

At present, the relative velocity method and the lever analogy are widely applied to analyze the AT from the kinematic points of view. The lever analogy employs the equivalent lever diagram which is more intuitive and is beneficial to the arrangement of gear ratios. In the present study, the lever analogy is applied to analyze the proposed AT mechanisms. Figure 10 illustrates the equivalent lever diagram of the proposed AT mechanism.

Figure 10 indicates that there are six shifting elements in the proposed AT mechanism, where two of them should contact simultaneously to get a certain gear ratio.
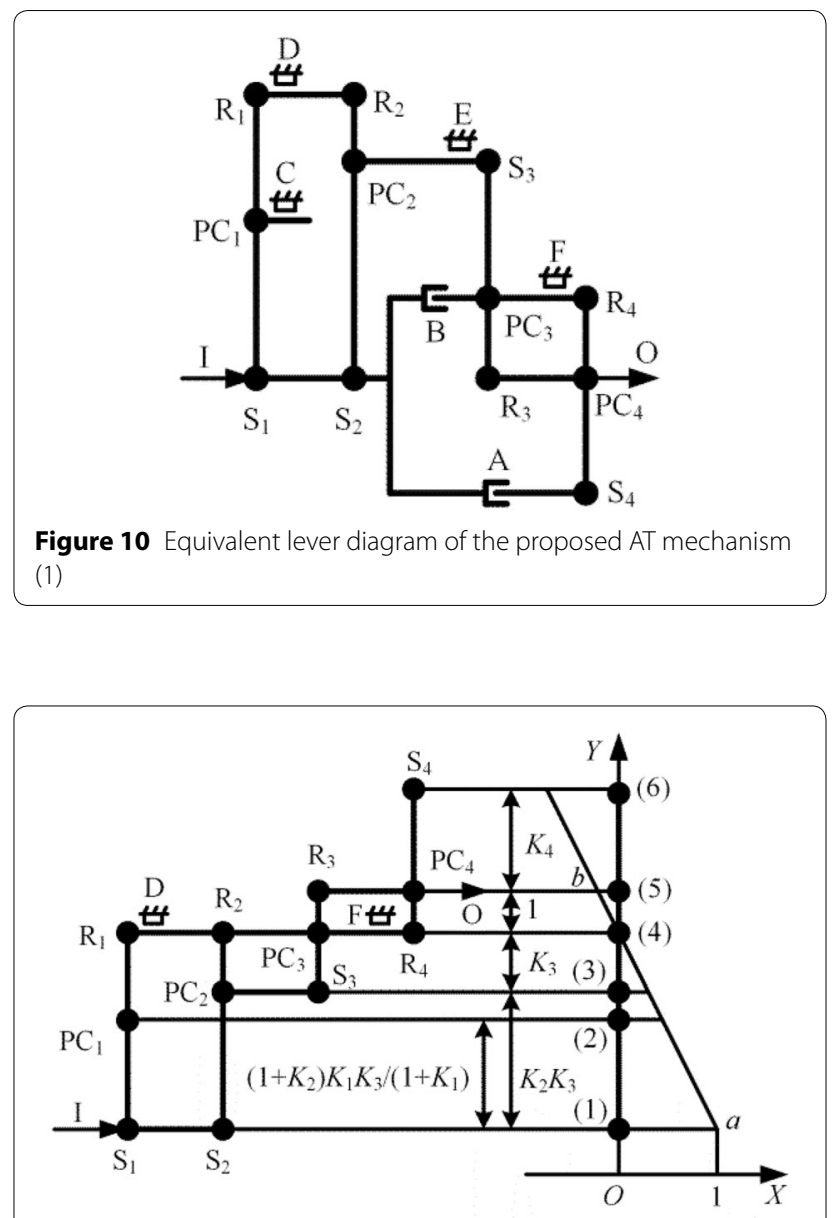

Figure 11 Equivalent lever speed diagram when $D$ and $F$ engaged

Considering the practical application of the AT system, for each PGS, the number of engaged brakes should not exceed one. Meanwhile, there are ten combination modes of clutches and brakes in the proposed AT mechanism. The characteristic parameters of four PGSs, which is equal to the ratio between teeth number of the ring gear and sun gear, are presented by $K_{n}(n=1,2,3,4)$.

\subsubsection{Combination Mode 1: $D$ and F Engaged}

In this mode, components $R_{1} R_{2}$ and $P C_{3} R_{4}$ are connected to the housing, while brakes $\mathrm{D}$ and $\mathrm{F}$ are engaged. Meanwhile, $R_{1} R_{2}$ and $P C_{3} R_{4}$ are stationary so that the corresponding velocities are 0 . By overlapping the fulcrums with the same speed into a fulcrum, the AT mechanism is transformed into an equivalent lever with six fulcrums as shown in Figure 11.

Fulcrums (1), (2) and (3) denote the input member $\mathrm{S}_{1} \mathrm{~S}_{2}$, member $\mathrm{PC}_{1}$ and the component $\mathrm{PC}_{2} \mathrm{~S}_{3}$, respectively. Moreover, fulcrums (4), (5) and (6) represent component $\mathrm{R}_{1} \mathrm{R}_{2} \mathrm{PC}_{3} \mathrm{R}_{4}$, output member $\mathrm{R}_{3} \mathrm{PC}_{4}$ and the member $\mathrm{S}_{4}$, 
respectively. The rectangular coordinate system $O-X Y$ is established as shown in Figure 11. The axis of the equivalent lever with six fulcrums is considered as the $Y$-axis, where the positive direction is from fulcrum (1) to fulcrum (6). Select an arbitrary point below the fulcrum (1) on the $Y$-axis as the origin point $O$, and then the straight line perpendicular to the $Y$-axis passing from the origin point $O$ is considered as the $X$-axis. In this case, the right direction is the positive direction.

The fulcrums on the $Y$-axis represent components of the EGT. For convenience of calculation, it is assumed that the length between fulcrum (4) and (5) is $1 \mathrm{~mm}$. Subsequently, the length between other fulcrums can be obtained and presented by the characteristic parameters $K_{n}(n=1,2,3,4)$.

The $X$-axis represents the rotational speed of each component. For convenience of calculation, it is assumed that the rotational speed of the input member (i.e., fulcrum (1)) is $1 \mathrm{r} / \mathrm{min}$, and the coordinate of point $a$ is $(1, Y(1))$. Moreover, the rotational speed of the fixed member (i.e., fulcrum (4)) is set to $0 \mathrm{r} / \mathrm{min}$. Then the speed line $a b$ of the AT mechanism at this time is obtained by connecting point $(1, Y(1))$ to point $(0, Y(4))$. Point $b$ is the intersection of the speed line and the horizontal line passing the fulcrum (5). $X$-coordinates of the speed line intersections with horizontal lines passing fulcrums represent the rotational speeds of the components represented by fulcrums. The positive and the negative signs represent the same and the opposite rotating direction of the components compared to the input member, respectively. For example, the $X$-coordinate of the $b$ point is negative, indicating that the rotating direction of the output member $\mathrm{R}_{3} \mathrm{PC}_{4}$ is opposite to that of the input member.

According to the basic properties of similar triangles, expressions of the rotational speed can be obtained for all components. For example, the triangle consisting of fulcrums (4), (5) and point $b$ is similar to the triangle consisting of fulcrums (4), (1) and point $a$. The $X$-coordinate of the $b$ point, namely the rotational speed of the output member $\mathrm{R}_{3} \mathrm{PC}_{4}$, can be derived accordingly:

$$
\begin{aligned}
& \frac{n_{\mathrm{R}_{3} \mathrm{PC}_{4}}}{n_{\mathrm{S}_{1} \mathrm{~S}_{2}}}=\frac{x_{b}}{x_{a}}=-\frac{l_{45}}{l_{14}}=-\frac{1}{\left(1+K_{2}\right) K_{3}}, \\
& n_{\mathrm{R}_{3} \mathrm{PC}_{4}}=x_{b}=-\frac{1}{\left(1+K_{2}\right) K_{3}},
\end{aligned}
$$

Table 1 Rotational speed of each component when $D$ and $F$ engaged $(r / m i n)$

\begin{tabular}{lcccccc}
\hline Component & $\mathbf{S}_{\mathbf{1}} \mathbf{S}_{\mathbf{2}}$ & $\mathbf{P C}_{\mathbf{1}}$ & $\mathbf{P C}_{\mathbf{2}} \mathbf{S}_{\mathbf{3}}$ & $\mathbf{R}_{\mathbf{1}} \mathbf{R}_{\mathbf{2}} \mathbf{P C}_{\mathbf{3}} \mathbf{R}_{\mathbf{4}}$ & $\mathbf{R}_{\mathbf{3}} \mathbf{P C}$ & $\mathbf{S}_{\mathbf{4}}$ \\
\hline Rotational speed & 1 & $\frac{1}{1+K_{1}}$ & $\frac{1}{1+K_{2}}$ & 0 & $-\frac{1}{\left(1+K_{2}\right) K_{3}}$ & $-\frac{1+K_{4}}{\left(1+K_{2}\right) K_{3}}$ \\
\hline
\end{tabular}
the input member $\mathrm{S}_{1} \mathrm{~S}_{2}$ and the output member $\mathrm{R}_{3} \mathrm{PC}_{4}$, respectively. Moreover, $x_{b}$ and $x_{a}$ are $X$-coordinate of $b$ and $a$ points, respectively. Finally, $l_{i j}(i, j=1,2, \ldots, 6)$ denotes the distance between fulcrums $(i)$ and $(j)$.

Similarly, the rotational speed of other components can be obtained. Calculated speeds are presented in Table 1.

$X$-coordinates of points $a$ and $b$ represent the rotational speed of the input and the output members, respectively. Based on the defined parameters, the gear ratio can be mathematically expressed as follows:

$$
i_{\mathrm{DF}}=\frac{n_{\mathrm{S}_{1} \mathrm{~S}_{2}}}{n_{\mathrm{R}_{3} \mathrm{PC}_{4}}}=-\left(1+K_{2}\right) K_{3},
$$

where $i_{\mathrm{DF}}$ denotes the gear ratio of the AT mechanism when $D$ and $F$ are engaged.

\subsubsection{Combination Mode 2: $C$ and F Engaged}

In this mode, the member $\mathrm{PC}_{1}$ and component $\mathrm{PC}_{3} \mathrm{R}_{4}$ are connected to the housing when brakes $C$ and $F$ are engaged. Meanwhile, $\mathrm{PC}_{1}$ and $\mathrm{PC}_{3} \mathrm{R}_{4}$ are stationary so that their speeds are set to 0 . Figure 12 illustrates the equivalent lever with six fulcrums transformed by the AT mechanism.

In this figure, the fulcrums (1), (2) and (3) denote the input member $S_{1} S_{2}$, member $S_{4}$ and output member (6) represent components $\mathrm{PC}_{1} \mathrm{PC}_{3} \mathrm{R}_{4}, \mathrm{PC}_{2} \mathrm{~S}_{3}$ and $\mathrm{R}_{1} \mathrm{R}_{2}$, respectively. Figure 12 indicates that the instantaneous speed line $a b$ of the AT mechanism can be obtained by connecting the coordinates $(1, Y(1))$ and $(0, Y(4))$.

Then expressions of the rotational speeds can be obtained for the components through the basic

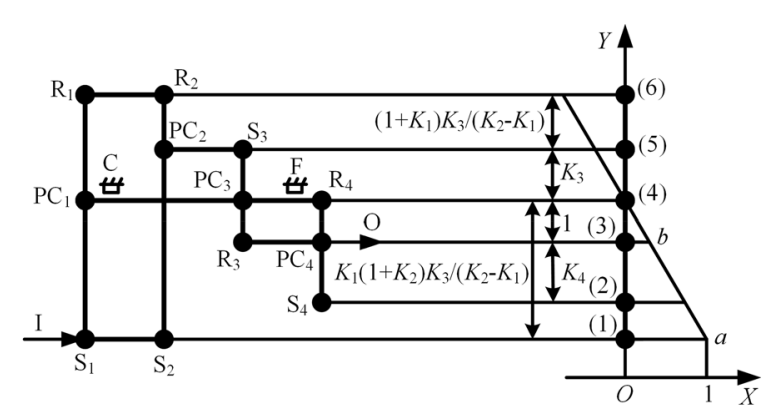

Figure 12 Equivalent lever speed diagram when $C$ and $F$ engaged where $n_{\mathrm{S}_{1} \mathrm{~S}_{2}}$ and $n_{\mathrm{R}_{3} \mathrm{PC}_{4}}$ denote the rotational speed of $\mathrm{R}_{3} \mathrm{PC}_{4}$, respectively. Moreover, fulcrums (4), (5) and 
properties of similar triangles. Table 2 illustrates the calculated results.

Based on the defined parameters, the gear ratio in this mode can be mathematically expressed as the following:

$$
i_{\mathrm{CF}}=\frac{n_{\mathrm{S}_{1} \mathrm{~S}_{2}}}{n_{\mathrm{R}_{3} \mathrm{PC}}}=\frac{\left(1+K_{2}\right) K_{1} K_{3}}{K_{2}-K_{1}},
$$

where $i_{\mathrm{CF}}$ denotes the gear ratio of the AT mechanism when $C$ and $F$ are engaged.

\subsubsection{Combination Mode 3-6: A Engaged}

In this mode, the clutch $A$ is engaged and member $S_{4}$ and component $S_{1} S_{2}$ are connected to each other. Figure 13 illustrates the equivalent lever with six fulcrums transformed by the AT mechanism.

Figure 13 indicates that fulcrums (1), (2) and (3) denote input member $\mathrm{S}_{1} \mathrm{~S}_{2} \mathrm{~S}_{4}$, output member $\mathrm{R}_{3} \mathrm{PC}_{4}$ and component $\mathrm{PC}_{3} \mathrm{R}_{4}$, respectively. Moreover, fulcrums (4), (5) and
(6) represent member $\mathrm{PC}_{1}$, component $\mathrm{PC}_{2} \mathrm{~S}_{3}$ and component $R_{1} R_{2}$, respectively.

There are four modes corresponding for engagements of different brakes. Therefore, four different gear ratios can be obtained accordingly.

In Figure 13, lines $a_{1} b_{1}$ and $a_{2} b_{2}$ represent the speed lines of the AT mechanism when brakes F and C, respectively are engaged. Moreover, $a_{3} b_{3}$ and $a_{4} b_{4}$ denote the speed lines of the AT mechanism when brakes E and D, respectively are engaged. Table 3 shows that the expressions of the rotational speeds for components when different brakes are engaged can be obtained by the basic properties of the similar triangle.

Component $\mathrm{S}_{1} \mathrm{~S}_{2} \mathrm{~S}_{4}$ is the input member, while $\mathrm{R}_{3} \mathrm{PC}_{4}$ is the output member. According to the definition of the gear ratio, the expressions are described as follows.

Gear ratio expression when brake $F$ is engaged is described as follows:

Table 2 Rotational speed of each component when $C$ and $F$ engaged ( $r / m i n)$

\begin{tabular}{lllllll}
\hline Component & $\mathbf{S}_{\mathbf{1}} \mathbf{S}_{\mathbf{2}}$ & $\mathbf{S}_{\mathbf{4}}$ & $\mathbf{R}_{\mathbf{3}} \mathbf{P} \mathbf{C}_{\mathbf{4}}$ & $\mathbf{P C}_{\mathbf{1}} \mathbf{P} \mathbf{C}_{\mathbf{3}} \mathbf{R}_{\mathbf{4}}$ & $\mathbf{P C}_{\mathbf{2}} \mathbf{S}_{\mathbf{3}}$ & $\mathbf{R}_{\mathbf{1}} \mathbf{R}_{\mathbf{2}}$ \\
\hline Rotational speed & 1 & $\frac{\left(1+K_{4}\right)\left(K_{2}-K_{1}\right)}{\left(1+K_{2}\right) K_{1} K_{3}}$ & $\frac{K_{2}-K_{1}}{\left(1+K_{2}\right) K_{1} K_{3}}$ & 0 & $-\frac{K_{2}-K_{1}}{\left(1+K_{2}\right) K_{1}}$ & $-\frac{1}{K_{1}}$ \\
\hline
\end{tabular}
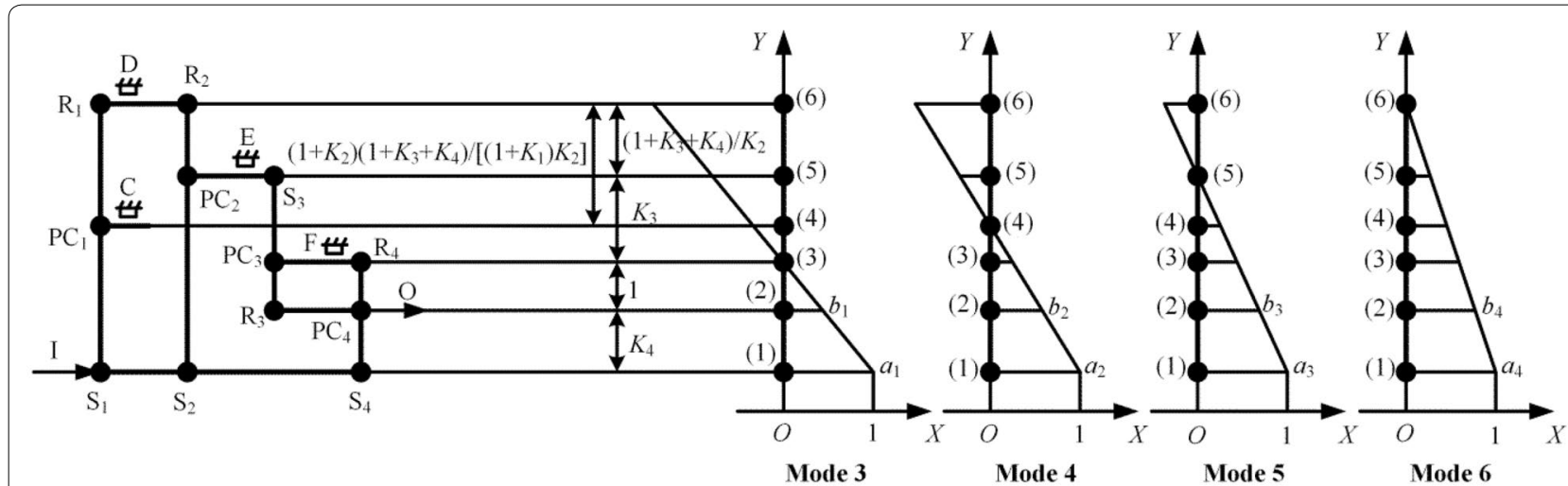

Figure 13 Equivalent lever speed diagram when the clutch A engaged

Table 3 Rotational speed of each component when A engaged ( $r / m i n)$

\begin{tabular}{|c|c|c|c|c|}
\hline & Mode 3 & Mode 4 & Mode 5 & Mode 6 \\
\hline $\mathrm{S}_{1} \mathrm{~S}_{2} \mathrm{~S}_{4}$ & 1 & 1 & 1 & 1 \\
\hline $\mathrm{R}_{3} \mathrm{PC}_{4}$ & $\frac{1}{1+K_{4}}$ & $1-\frac{\left(1+K_{1}\right) K_{2} K_{4}}{\left(1+K_{3}+K_{4}\right)\left(1+K_{2}\right) K_{1}}$ & $\frac{1+K_{3}}{1+K_{3}+K_{4}}$ & $1-\frac{K_{2} K_{4}}{\left(1+K_{3}+K_{4}\right)\left(1+K_{2}\right)}$ \\
\hline $\mathrm{PC}_{3} \mathrm{R}_{4}$ & 0 & $1-\frac{\left(1+K_{1}\right)\left(1+K_{4}\right) K_{2}}{\left(1+K_{3}+K_{4}\right)\left(1+K_{2}\right) K_{1}}$ & $\frac{K_{3}}{1+K_{3}+K_{4}}$ & $\frac{1+K_{3}+K_{4}+K_{2} K_{3}}{\left(1+K_{3}+K_{4}\right)\left(1+K_{2}\right)}$ \\
\hline $\mathrm{PC}_{1}$ & $1-\frac{\left(1+K_{3}+K_{4}\right)\left(1+K_{2}\right) K_{1}}{\left(1+K_{1}\right)\left(1+K_{4}\right) K_{2}}$ & 0 & $\frac{K_{2}-K_{1}}{\left(1+K_{1}\right) K_{2}}$ & $\frac{1}{1+K_{1}}$ \\
\hline $\mathrm{PC}_{2} \mathrm{~S}_{3}$ & $-\frac{K_{3}}{1+K_{4}}$ & $-\frac{K_{2}-K_{1}}{\left(1+K_{2}\right) K_{1}}$ & 0 & $\frac{1}{1+K_{2}}$ \\
\hline $\mathrm{R}_{1} \mathrm{R}_{2}$ & $\frac{1+K_{3}+K_{4}+K_{2} K_{3}}{\left(1+K_{4}\right) K_{2}}$ & $-\frac{1}{K_{1}}$ & $-\frac{1}{K_{2}}$ & 0 \\
\hline
\end{tabular}




$$
i_{\mathrm{AF}}=\frac{n_{\mathrm{S}_{1} \mathrm{~S}_{2} \mathrm{~S}_{4}}}{n_{\mathrm{R}_{3} \mathrm{PC}_{4}}}=1+K_{4} .
$$

Gear ratio expression when brake $C$ is engaged is described as follows:

$$
i_{\mathrm{AC}}=\frac{n_{\mathrm{S}_{1} \mathrm{~S}_{2} \mathrm{~S}_{4}}}{n_{\mathrm{R}_{3} \mathrm{PC}_{4}}}=\frac{\left(1+K_{3}+K_{4}\right)\left(1+K_{2}\right) K_{1}}{\left(\left(1+K_{2}\right)\left(1+K_{3}\right)+K_{4}\right) K_{1}-K_{2} K_{4}} .
$$

Gear ratio expression when brake $\mathrm{E}$ is engaged is described as follows:

$$
i_{\mathrm{AE}}=\frac{n_{\mathrm{S}_{1} \mathrm{~S}_{2} \mathrm{~S}_{4}}}{n_{\mathrm{R}_{3} \mathrm{PC}_{4}}}=1+\frac{K_{4}}{1+K_{3}} .
$$

Gear ratio expression when brake D is engaged is described as follows:

$$
i_{\mathrm{AD}}=\frac{n_{\mathrm{S}_{1} \mathrm{~S}_{2} \mathrm{~S}_{4}}}{n_{\mathrm{R}_{3} \mathrm{PC}}}=\frac{\left(1+K_{2}\right)\left(1+K_{3}+K_{4}\right)}{1+K_{2}+K_{3}+K_{4}+K_{2} K_{3}},
$$

where $i_{\mathrm{AF}}, i_{\mathrm{AC}}, i_{\mathrm{AE}}$ and $i_{\mathrm{AD}}$ denote the gear ratio of the $\mathrm{AT}$ mechanism when $A$ and $F$ are engaged, gear ratio when $A$ and $C$ are engaged, gear ratio when $A$ and $E$ are engaged and the gear ratio when $\mathrm{A}$ and $\mathrm{D}$ are engaged, respectively. Moreover, $n_{S_{1} S_{2} S_{4}}$ represents the rotational speed of the input member $S_{1} S_{2} S_{4}$.

\subsubsection{Combination Mode 7-9: B Engaged}

In this mode, the clutch $B$ is engaged and components $\mathrm{PC}_{3} \mathrm{R}_{4}$ and $\mathrm{S}_{1} \mathrm{~S}_{2}$ are connected to each other. Figure 14 shows the equivalent lever with six fulcrums transformed by the AT mechanism.

It should be indicated that the fulcrums (1), (2) and (3) represent the member $\mathrm{S}_{4}$, output member $\mathrm{R}_{3} \mathrm{PC}_{4}$ and input member $\mathrm{S}_{1} \mathrm{~S}_{2} \mathrm{PC}_{3} \mathrm{R}_{4}$, respectively. Moreover, the fulcrums (4), (5) and (6) represent the member $\mathrm{PC}_{1}$, component $\mathrm{PC}_{2} \mathrm{~S}_{3}$, and component $\mathrm{R}_{1} \mathrm{R}_{2}$, respectively.

Different gear ratios are obtained by engaging different brakes. Figure 14 shows that the lines $a_{1} b_{1}, a_{2} b_{2}$ and $a_{3} b_{3}$ represent the speed lines of the AT mechanism when brakes $D, E$ and $C$, respectively are engaged. Moreover, Table 4 shows that the expressions of the rotational speeds for the components when different brakes are engaged can be obtained by the basic properties of the similar triangle.

Component $\mathrm{S}_{1} \mathrm{~S}_{2} \mathrm{PC}_{3} \mathrm{R}_{4}$ is the input member, while $\mathrm{R}_{3} \mathrm{PC}_{4}$ is the output member. According to the definition of the gear ratio, the expressions can be obtained as follows.

When the brake $\mathrm{D}$ is engaged, the corresponding gear ratio can be expressed as the following:

$$
i_{\mathrm{BD}}=\frac{n_{\mathrm{S}_{1} \mathrm{~S}_{2} \mathrm{PC}_{3} \mathrm{R}_{4}}}{n_{\mathrm{R}_{3} \mathrm{PC}_{4}}}=\frac{\left(1+K_{2}\right) K_{3}}{K_{2}+K_{3}\left(1+K_{2}\right)} .
$$

When the brake $\mathrm{E}$ is engaged, the mathematical expression for the gear ratio is:

Table 4 Rotational speed of each component when B engaged $(r / \mathrm{min})$

\begin{tabular}{llll}
\hline & Mode 7 & Mode 8 & Mode 9 \\
\hline $\mathrm{S}_{4}$ & $\frac{\left(1+K_{4}\right) K_{2}}{\left(1+K_{2}\right) K_{3}}+1$ & $\frac{1+K_{4}}{K_{3}}+1$ & $1+\frac{\left(1+K_{1}\right)\left(1+K_{4}\right) K_{2}}{\left(1+K_{2}\right) K_{1} K_{3}}$ \\
$\mathrm{R}_{3} \mathrm{PC}_{4}$ & $\frac{K_{2}}{\left(1+K_{2}\right) K_{3}}+1$ & $\frac{1}{K_{3}}+1$ & $1+\frac{\left(1+K_{1}\right) K_{2}}{\left(1+K_{2}\right) K_{1} K_{3}}$ \\
$\mathrm{~S}_{1} \mathrm{~S}_{2} \mathrm{PC}_{3} \mathrm{R}_{4}$ & 1 & 1 & 1 \\
$\mathrm{PC}_{1}$ & $\frac{1}{1+K_{1}}$ & $\frac{K_{2}-K_{1}}{\left(1+K_{1}\right) K_{2}}$ & 0 \\
$\mathrm{PC}_{2} \mathrm{~S}_{3}$ & $\frac{1}{1+K_{2}}$ & 0 & $-\frac{K_{2}-K_{1}}{\left(1+K_{2}\right) K_{1}}$ \\
$\mathrm{R}_{1} \mathrm{R}_{2}$ & 0 & $-\frac{1}{K_{2}}$ & $-\frac{1}{K_{1}}$ \\
\hline
\end{tabular}

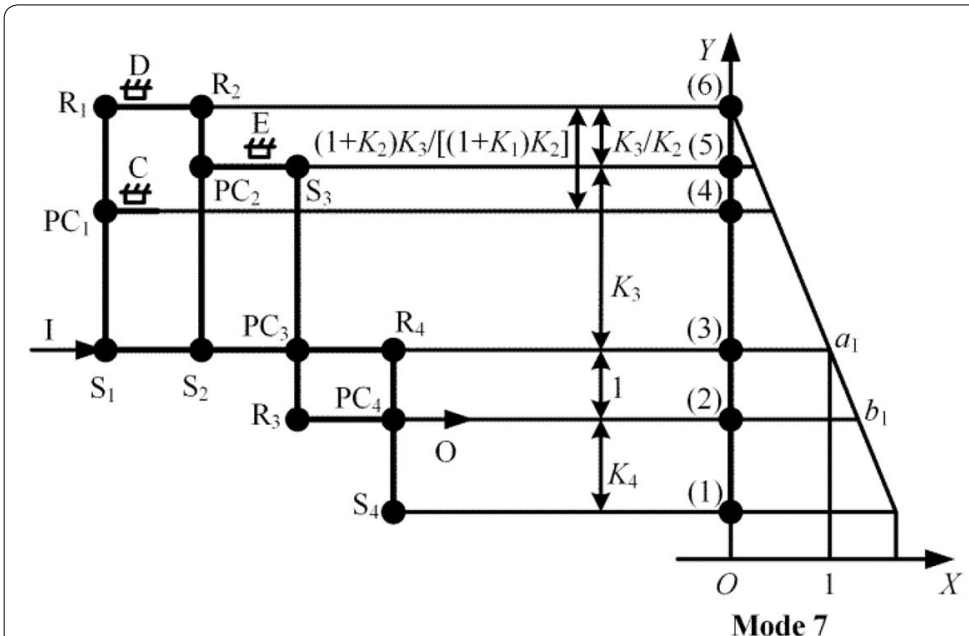

Figure 14 Equivalent lever speed diagram when clutch B engaged
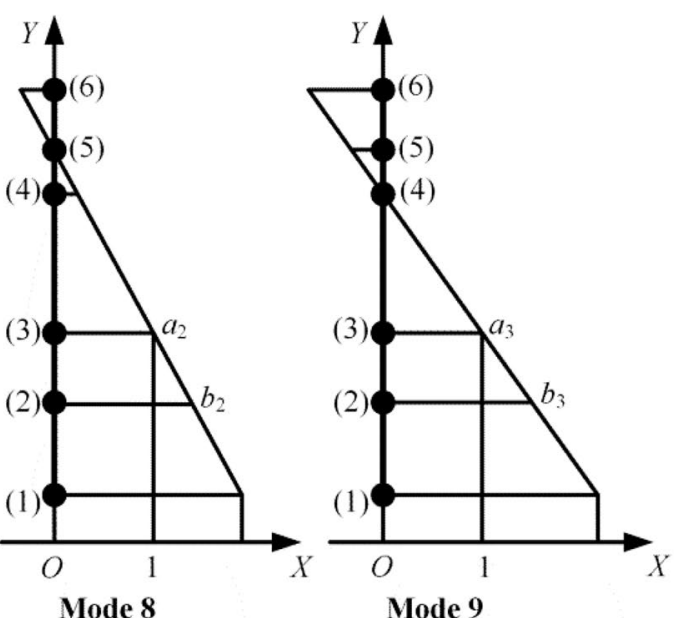


$$
i_{\mathrm{BE}}=\frac{n_{\mathrm{S}_{1} \mathrm{~S}_{2} \mathrm{PC}_{3} \mathrm{R}_{4}}}{n_{\mathrm{R}_{3} \mathrm{PC}_{4}}}=\frac{K_{3}}{1+K_{3}} .
$$

When the brake $\mathrm{C}$ is engaged, the expression for the gear ratio is in the form below:

$$
i_{\mathrm{BC}}=\frac{n_{\mathrm{S}_{1} \mathrm{~S}_{2} \mathrm{PC}_{3} \mathrm{R}_{4}}}{n_{\mathrm{R}_{3} \mathrm{PC}_{4}}}=\frac{\left(1+K_{2}\right) K_{1} K_{3}}{\left(1+K_{1}\right) K_{2}+\left(1+K_{2}\right) K_{1} K_{3}},
$$

where $i_{\mathrm{BD}}, i_{\mathrm{BE}}$ and $i_{\mathrm{BC}}$ denote the gear ratio of the AT mechanism when $\mathrm{B}$ and $\mathrm{D}, \mathrm{B}$ and $\mathrm{E}$, and $\mathrm{B}$ and $\mathrm{C}$ are

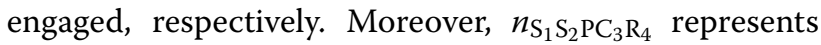
the rotational speed of the input member $\mathrm{S}_{1} \mathrm{~S}_{2} \mathrm{PC}_{3} \mathrm{R}_{4}$.

\subsubsection{Combination Mode 10: $A$ and $B$ Engaged}

In this mode, components $\mathrm{PC}_{3} \mathrm{R}_{4}, \mathrm{~S}_{1} \mathrm{~S}_{2}$ and member $\mathrm{S}_{4}$ are connected to each other when clutches $\mathrm{A}$ and $\mathrm{B}$ are engaged together. Meanwhile, the $\mathrm{PGS}_{4}$ moves alone to form an atresia, which transmits the input movement to the output member directly, realizing the direct gear. Figure 15 indicates that connecting the coordinates $(1, Y(1))$ and $(1, Y(3))$ and the speed line $a_{1} a_{2}$ of the AT mechanism can be obtained.

In this mode, rotational speeds of all components are the same, which is equal to that of the input member, namely, $1 \mathrm{r} / \mathrm{min}$. This can be mathematically expressed as the following:

$$
n_{\mathrm{S}_{1} \mathrm{~S}_{2} \mathrm{~S}_{4}}=n_{\mathrm{R}_{3} \mathrm{PC}_{4}}=n_{\mathrm{PC}_{3} \mathrm{R}_{4}}=n_{\mathrm{PC}_{1}}=n_{\mathrm{PC}_{2} \mathrm{~S}_{3}}=n_{\mathrm{R}_{1} \mathrm{R}_{2}}=1 \text {. }
$$

$X$-coordinates of points $a_{1}$ and $a_{2}$ represent the rotational speed of the input member at the same time. Moreover, the $X$-coordinate of point $b$ represents the rotational speed of the output member. Then, the gear ratio can be obtained as follows:

$$
i_{\mathrm{AB}}=\frac{n_{\mathrm{S}_{1} \mathrm{~S}_{2} \mathrm{~S}_{4}}}{n_{\mathrm{R}_{3} \mathrm{PC}_{4}}}=1,
$$

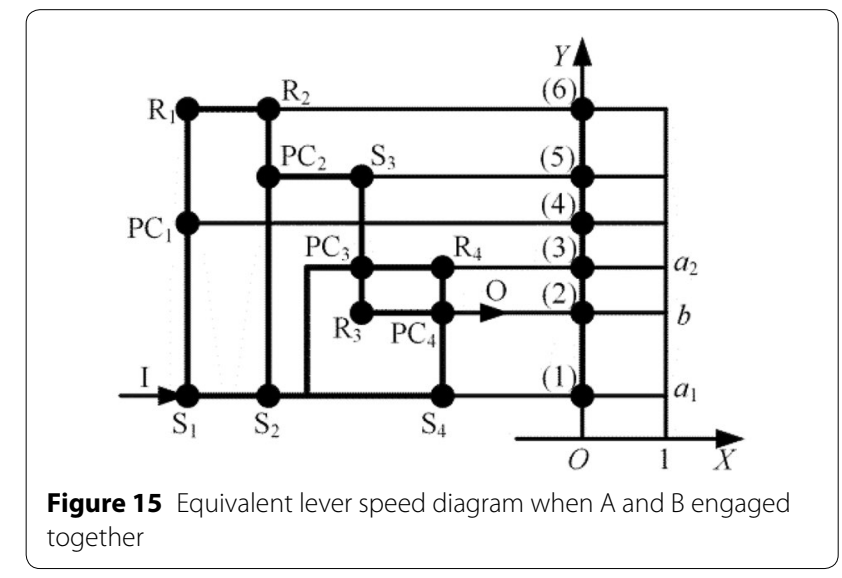

Figure 15 Equivalent lever speed diagram when $A$ and $B$ engaged together where $i_{\mathrm{AB}}$ and $n_{\mathrm{S}_{1} \mathrm{~S}_{2} \mathrm{~S}_{4}}$ denote the gear ratio of the AT mechanism when $A$ and $B$ are engaged and the rotational speed of the input member $S_{1} S_{2} S_{4}$, respectively.

\subsection{Mechanical Analysis}

The mechanical analysis of the AT mechanism refers to calculating torques of gear meshing points, including the external torque of the EGT mechanism and the internal torque of PGSs. As the key part of the AT mechanism, the torque applied on members of the mechanical transmission remarkably affects the working performance and service life [48]. In order to perform the analysis, it is assumed that there is no friction in the AT mechanism, and it has a uniform motion. The mechanical analysis of the AT mechanism can be simplified into the problem of equilibrium equations of parallel forces, which can be resolved by the lever analogy method.

If a member is applied to transmit power, the PGS including the member is said to be active. Take the force condition under reverse gear as example. Then, two PGSs are active, while the other two PGSs do not participate in the power transmission when the brakes $D$ and $F$ are engaged. Figure 16 shows the force condition. It should be indicated that the input and output members are the sun gear $S_{2}$ and the ring gear $R_{3}$, respectively. Moreover, the fixed members are the ring gear $\mathrm{R}_{2}$ and the carrier $\mathrm{PC}_{3}$.

In Figure $16, T_{\mathrm{I}}, T_{\mathrm{O}}$ and $T_{\mathrm{b}}$ denote the input torque, output torque and the brake torque, respectively.

\subsubsection{External Torque Analysis}

Generally, the input torque $T_{\mathrm{I}}$ is given and acts on the input member $\mathrm{S}_{2}$. Moreover, the output torque $T_{\mathrm{O}}$ acting on the output member $R_{3}$ can be calculated in the form below, based on the gear ratio obtained in Section 3.1.

$$
T_{\mathrm{O}}=-i_{\mathrm{DF}} T_{\mathrm{I}}=\left(1+K_{2}\right) K_{3} T_{\mathrm{I}} .
$$

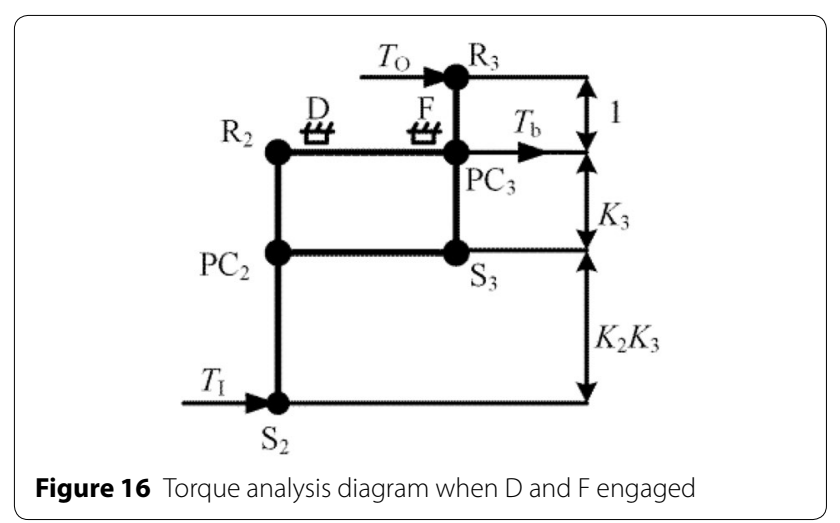


The brake torque $T_{\mathrm{b}}$ acting on fixed members $\mathrm{R}_{2}$ and $\mathrm{PC}_{3}$ can be calculated in accordance with the balance of external torques from the horizontal direction:

$$
T_{\mathrm{b}}=-T_{\mathrm{I}}-T_{\mathrm{O}}=-\left(1+K_{3}+K_{2} K_{3}\right) T_{\mathrm{I}} .
$$

\subsubsection{Internal Torque Analysis}

The internal torque of PGS refers to the applied torque by the planet gear to central gears meshing with it or the carrier supporting it. According to the equilibrium equation of torques applied by central gears and carrier on the planet gear in a PGS, the following correlation holds between torques:

$$
\frac{T_{\mathrm{S}}}{1}=\frac{T_{\mathrm{R}}}{K}=\frac{T_{\mathrm{PC}}}{-(1+K)} .
$$

It should be indicated that the member with the certain external torque and only one internal torque is initially analyzed. In fact, this specific member is only involved in the motion of one PGS. According to the Newton's third law, the internal torque of the member is equal in magnitude and opposite in direction compared with the external torque acting on it.

Figure 16 shows that the input member $\mathrm{S}_{2}$ only participates in the motion of $\mathrm{PGS}_{2}$. Then, the internal torque of $\mathrm{S}_{2}$ can be obtained as the following:

$$
T_{\mathrm{S}_{2}}=-T_{\mathrm{I}} \text {. }
$$

Based on Eq. (19), the internal torque of $R_{2}$ can be obtained as:

$$
T_{\mathrm{R}_{2}}=K_{2} T_{\mathrm{S}_{2}}=-K_{2} T_{\mathrm{I}}
$$

Then, the internal torque of $\mathrm{PC}_{2}$ can be expressed as the following:

$$
T_{\mathrm{PC}_{2}}=-T_{\mathrm{S}_{2}}-T_{\mathrm{R}_{2}}=\left(1+K_{2}\right) T_{\mathrm{I}} .
$$

On the other hand, the output member $R_{3}$ only participates in the motion of $\mathrm{PGS}_{3}$ so that the internal torque of $R_{3}$ can be obtained as follows:

$$
T_{\mathrm{R}_{3}}=-T_{\mathrm{O}}=-\left(1+K_{2}\right) K_{3} T_{\mathrm{I}} .
$$

Based on Eq. (19), the internal torque of $S_{3}$ can be expressed as:

$$
T_{\mathrm{S}_{3}}=\frac{1}{K_{3}} T_{\mathrm{R}_{3}}=-\left(1+K_{2}\right) T_{\mathrm{I}} .
$$

Then, the internal torque of $\mathrm{PC}_{3}$ can be obtained in the form below:

$$
T_{\mathrm{PC}_{3}}=-T_{\mathrm{S}_{3}}-T_{\mathrm{R}_{3}}=\left(1+K_{2}\right)\left(1+K_{3}\right) T_{\mathrm{I}} .
$$

The analysis process of other gears is similar to that of the reverse gear. Therefore, they are not discussed one by one in this article. Under the condition of given input torque or certain load, the torque of each member can be calculated to check the working state of members and evaluate the performance and service life.

\subsection{Power Flow Analysis}

For a certain combination mode, the path of the power transmission inside the AT mechanism can be described clearly by the power flow analysis, which is beneficial for the observation of the circulating power and plays an important role in the accurate efficiency evaluation [25]. The rotational speed and internal torque of each member are derived based on the kinematics and mechanical analysis. Then, the power transmitted by each member is described by the following equation:

$$
P_{\mathrm{X}}=T_{\mathrm{X}} \frac{2 \pi n_{\mathrm{X}}}{60}=\frac{\pi}{30} T_{\mathrm{X}} n_{\mathrm{X}},
$$

where $P_{\mathrm{X}}, T_{\mathrm{X}}$ and $n_{\mathrm{X}}$ denote the power, internal torque of the member $\mathrm{X}$ and the rotational speed of the member $\mathrm{X}$, respectively.

Assuming the direction of the input torque and input rotational speed as the positive direction, the power direction is judged by the following rules:

If $P_{\mathrm{X}}>0$, power flows into the member $\mathrm{X}$ so that the member $\mathrm{X}$ is the driven member;

If $P_{\mathrm{X}}<0$, power flows out of the member $\mathrm{X}$ so that the member $\mathrm{X}$ is the driving member;

If $P_{\mathrm{X}}=0$, power flows through the member $\mathrm{X}$.

For members in a PGS, the power flows from the driving member to the driven member. Moreover, for the members connected by ICs or shifting elements, the power flows from the driven member to the driving member. It should be indicated that arrows are applied in the equivalent lever diagram to indicate the direction of the power. Then, the power flow diagrams under each gear ratio can be obtained to express the paths of the power transmission.

If the power transmitted through any component exceeds the input power, circulating power occurs. The circulating power is harmful and reduces the transmission efficiency, especially when the circulating power is too high. Therefore, the circulating power should be considered at the design stage of AT mechanisms.

\subsection{Transmission Efficiency Analysis}

The transmission efficiency of the AT mechanism is an important parameter to evaluate the performance of the mechanism. For the convenience of calculation, the following assumptions are made in the analysis [42]: 
(1) Only the gear meshing loss is considered and other losses, such as bearing loss and splash loss are ignored.

(2) Assume that there is no loss caused by the implicated motion. Moreover, implicated motion does not cause the gear mesh transmission.

(3) Assume that the total transmission loss of the PGS is caused by the gear meshing loss in the relative motion. Moreover, the gear meshing loss caused by the relative motion is the same as the fix axle transmission.

Based on the abovementioned assumptions, the gear meshing loss is actually the torque loss caused by the friction at gear pairs. Therefore, the torque method is used to solve the transmission efficiency in the present study. The torque method is suitable for all structures of AT mechanisms and the derivation process is simple. The calculation equation is as follows:

$$
\eta=\frac{\hat{i}}{i}
$$

where $\eta$ denotes the transmission efficiency of the AT mechanism. Moreover, $i=f\left(K_{1}, K_{2}, \cdots, K_{n}\right)$ denotes the ideal gear ratio, where $n$ denotes the number of PGS in the mechanism. $\hat{i}=f\left(K_{1} \eta_{\mathrm{c}}^{x_{1}}, K_{2} \eta_{\mathrm{c}}^{x_{2}}, \cdots, K_{n} \eta_{\mathrm{c}}^{x_{n}}\right)$ denotes the real torque transformation, where $\eta_{\mathrm{c}}$ indicates the efficiency of the PGS when the carrier is fixed with a value of 0.97 . The value of $x_{m}(m=1,2, \ldots, n)$ involves the power flow directions of the $\mathrm{PGS}_{m}$ and it is calculated by the following equation:

$$
x_{m}=\operatorname{sign}\left(\frac{\partial \ln i}{\partial K_{m}}\right) .
$$

Namely, $x_{m}=+1$ when $\frac{\partial \ln i}{\partial K_{m}}>0$, while $x_{m}=-1$ when $\frac{\partial \ln i}{\partial K_{m}}<0$.

\subsection{Numerical Example and Comparative Analysis}

Considering the rationality of the radial size, the range of the characteristic parameter $K$ of single-planet PGS is generally $4 / 3-4$. In order to improve the shift comfort of AT mechanisms, the interval of gear ratios should be as small as possible in the range of 1.1-1.6. It should be indicated that the lower limit of the transmission efficiency of the forward gears is not less than 0.925. However, it is allowed not to be less than 0.87 for the rarely used gears, such as the first gear and reverse gear [49].

\subsubsection{Numerical Example}

In order to obtain a series of characteristic parameters and corresponding gear ratio sets, characteristic parameters of the 4 PGSs are considered as variables, while the variation range of characteristic parameters and the interval of gear ratios are considered as the cyclic interval and the constraint condition, respectively. Take one of the characteristic parameters sets as example, $K_{1}=1.4$, $K_{2}=3, K_{3}=1.4$ and $K_{4}=2.2$. Table 5 shows the gear ratios, interval of gear ratios and gear ranking of the AT mechanism.

Table 5 shows that the interval of gear ratios between each gear approaches the empirical value, and the characteristic parameters meet the application requirements.

Assume that the rotational speed of the input member is $1 \mathrm{r} / \mathrm{min}$ and the external torque applied to input member is $1 \mathrm{~N} \cdot \mathrm{m}$. Table 6 shows that by ignoring the power loss, the power passing through each member can be obtained based on Eq. (26).

The power flow diagrams under each gear ratio can be obtained according to the sign of powers shown in Table 6. Taking the reverse gear and 1st gear as examples, the power flow diagrams are shown in Figure 17.

It is observed that the circulating power occurs under the 1st gear. The value of the circulating power is equal to the power of member $\mathrm{S}_{2}$, namely, $0.875 \mathrm{~W}$, which is not too high. Therefore, the AT mechanism is still available.

The transmission efficiency of the AT mechanism can be calculated based on the torque method. Taking the reverse gear as example and according to Eqs. (6) and (28), the following equations are obtained:

$$
\begin{aligned}
& \frac{\partial \ln \left(-\left(1+K_{2}\right) K_{3}\right)}{\partial K_{2}}=\frac{1}{1+K_{2}}=\frac{1}{4}>0, \\
& \frac{\partial \ln \left(-\left(1+K_{2}\right) K_{3}\right)}{\partial K_{3}}=\frac{1}{K_{3}}=\frac{1}{1.4}>0 .
\end{aligned}
$$

According to Eqs. (28)-(30), it is calculated that $x_{2}=+1, x_{3}=+1$. Then, the real torque transformation can be obtained as the following:

Table 5 Gear ratios, interval of gear ratio and gear ranking of the novel AT mechanism (1)

\begin{tabular}{llll}
\hline Combination mode & Gear ratio & $\begin{array}{l}\text { Interval } \\
\text { of gear ratio }\end{array}$ & Gear \\
\hline Mode 1 & -5.60 & - & Reverse gear \\
Mode 2 & 4.90 & - & 1st gear \\
Mode 3 & 3.20 & 1.53 & 2nd gear \\
Mode 4 & 2.60 & 1.23 & 3rd gear \\
Mode 5 & 1.92 & 1.36 & 4th gear \\
Mode 6 & 1.56 & 1.23 & 5th gear \\
Mode 10 & 1 & 1.56 & 6th gear \\
Mode 7 & 0.65 & 1.54 & 7th gear \\
Mode 8 & 0.58 & 1.12 & 8th gear \\
Mode 9 & 0.52 & 1.12 & 9th gear \\
\hline
\end{tabular}


Table 6 Power passing through each member of the novel AT mechanism (1) (W)

\begin{tabular}{lllllllllllll}
\hline & S & PC1 & R1 & S2 & PC2 & R2 & S3 & PC3 & R3 & S4 & PC4 & R4 \\
\hline Reverse gear & - & - & - & -1 & 1 & 0 & -1 & 0 & 1 & - & - & - \\
1st gear & -1.875 & 0 & 1.875 & 0.875 & 1 & -1.875 & -1 & 0 & 1 & - & - & - \\
2nd gear & - & - & - & - & - & - & - & - & - & -1 & 1 & 0 \\
3rd gear & 0.665 & 0 & -0.665 & -0.310 & -0.355 & 0.665 & 0.355 & 0.315 & -0.670 & -1.355 & 1.670 & -0.315 \\
4th gear & - & - & - & - & - & - & 0 & 0.670 & -0.670 & -1 & 1.670 & -0.670 \\
5th gear & - & - & - & -0.186 & 0.186 & 0 & -0.186 & 0.856 & -0.670 & -0.814 & 1.670 & -0.856 \\
6th gear & - & - & - & - & - & - & - & - & - & -0.3125 & 1 & -0.6875 \\
7th gear & - & - & - & 0.116 & -0.116 & 0 & 0.116 & -1.116 & 1 & - & - & - \\
8th gear & - & - & - & - & - & - & 0 & -1 & 1 & - & - & - \\
9th gear & -0.199 & 0 & 0.199 & 0.093 & 0.106 & -0.199 & -0.106 & -0.894 & 1 & - & - & - \\
\hline
\end{tabular}

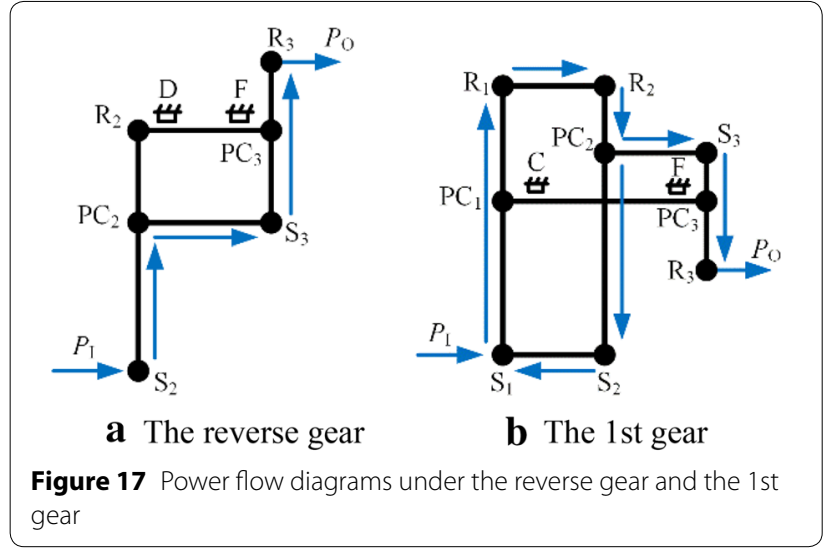

$$
\hat{i}=-\left(1+K_{2} \times 0.97^{x_{2}}\right) \times\left(K_{3} \times 0.97^{x_{3}}\right)=-5.31
$$

Then, the transmission efficiency is obtained as the following:

$$
\eta=\frac{\hat{i}}{i}=\frac{-5.31}{-5.6}=0.9482
$$

Similarly, the transmission efficiencies of other gears are calculated, and the calculation results are shown in Table 7.

Table 7 shows that the transmission efficiencies approach the empirical value. Therefore, the transmission efficiencies of the reverse gear, 1st gear and 3rd gear are a little lower.

Moreover, the kinematics and transmission efficiency of the other three novel AT mechanisms are analyzed. Tables 8, 9 and 10 show the gear ratios, interval of gear ratios and the transmission efficiency of each AT mechanism, respectively.
Table 7 Transmission efficiency under each gear

\begin{tabular}{ll}
\hline Gear & $\begin{array}{l}\text { Transmission } \\
\text { efficiency }\end{array}$ \\
\hline Reverse gear & 0.9482 \\
1st gear & 0.8879 \\
2nd gear & 0.9794 \\
3rd gear & 0.9333 \\
4th gear & 0.9774 \\
5th gear & 0.9821 \\
6th gear & 1 \\
7th gear & 0.9867 \\
8th gear & 0.9873 \\
9th gear & 0.9757 \\
\hline
\end{tabular}

Table 8 Gear ratios, interval of gear ratio and transmission efficiency of the novel AT mechanism (2)

\begin{tabular}{llll}
\hline Gear & Gear ratio & $\begin{array}{l}\text { Interval of } \\
\text { gear ratio }\end{array}$ & $\begin{array}{l}\text { Transmission } \\
\text { efficiency }\end{array}$ \\
\hline Reverse gear & -9.32 & - & 0.9355 \\
1st gear & 4.51 & - & 0.9286 \\
2nd gear & 2.90 & 1.56 & 0.9803 \\
3rd gear & 2.36 & 1.23 & 0.9510 \\
4th gear & 1.76 & 1.34 & 0.9794 \\
5th gear & 1.57 & 1.12 & 0.9808 \\
6th gear & 1 & 1.57 & 1 \\
7th gear & 0.64 & 1.56 & 0.9865 \\
8th gear & 0.60 & 1.07 & 0.9878 \\
9th gear & 0.53 & 1.13 & 0.9804 \\
\hline
\end{tabular}

\subsubsection{Comparative Analysis}

The gear ratios and intervals of gear ratios of the existing AT mechanisms are obtained from Refs. [38-40]. Moreover, the transmission efficiencies of the existing AT mechanisms are calculated based on the lever analogy 
Table 9 Gear ratios, interval of gear ratio and transmission efficiency of the novel AT mechanism (3)

\begin{tabular}{llll}
\hline Gear & Gear ratio & $\begin{array}{l}\text { Interval of } \\
\text { gear ratio }\end{array}$ & $\begin{array}{l}\text { Transmission } \\
\text { efficiency }\end{array}$ \\
\hline Reverse gear & -3.00 & - & 0.9333 \\
1st gear & 5.57 & - & 0.8867 \\
2nd gear & 3.30 & 1.69 & 0.9791 \\
3rd gear & 1.95 & 1.69 & 0.9643 \\
4th gear & 1.75 & 1.11 & 0.9845 \\
5th gear & 1.58 & 1.11 & 0.9713 \\
6th gear & 1 & 1.58 & 1 \\
7th gear & 0.80 & 1.25 & 0.9895 \\
8th gear & 0.67 & 1.19 & 0.9803 \\
9th gear & 0.55 & 1.22 & 0.9714 \\
\hline
\end{tabular}

Table 10 Gear ratios, interval of gear ratio and transmission efficiency of the novel AT mechanism (4)

\begin{tabular}{llll}
\hline Gear & Gear ratio & $\begin{array}{l}\text { Interval of } \\
\text { gear ratio }\end{array}$ & $\begin{array}{l}\text { Transmission } \\
\text { efficiency }\end{array}$ \\
\hline Reverse gear & -9.67 & - & 0.8774 \\
1st gear & 4.68 & - & 0.8731 \\
2nd gear & 2.8 & 1.67 & 0.9807 \\
3rd gear & 2.28 & 1.23 & 0.9350 \\
4th gear & 1.78 & 1.28 & 0.9655 \\
5th gear & 1.61 & 1.11 & 0.9695 \\
6th gear & 1 & 1.61 & 1 \\
7th gear & 0.6 & 1.67 & 0.9603 \\
8th gear & 0.57 & 1.05 & 0.9595 \\
9th gear & 0.5 & 1.14 & 0.9494 \\
\hline
\end{tabular}

Table 11 The gear ratios, interval of gear ratio and transmission efficiency of ZF 9HP

\begin{tabular}{llll}
\hline Gear & Gear ratio & $\begin{array}{l}\text { Interval of } \\
\text { gear ratio }\end{array}$ & $\begin{array}{l}\text { Transmission } \\
\text { efficiency }\end{array}$ \\
\hline Reverse gear & -3.80 & & 0.8960 \\
1st gear & 4.70 & & 0.9660 \\
2nd gear & 2.85 & 1.65 & 0.9738 \\
3rd gear & 1.90 & 1.49 & 0.9836 \\
4th gear & 1.38 & 1.38 & 0.9917 \\
5th gear & 1 & 1.38 & 1 \\
6th gear & 0.80 & 1.24 & 0.9855 \\
7th gear & 0.70 & 1.16 & 0.9878 \\
8th gear & 0.58 & 1.21 & 0.9778 \\
9th gear & 0.48 & 1.21 & 0.9736 \\
\hline
\end{tabular}

Table 12 The gear ratios, interval of gear ratio and transmission efficiency of Benz 9G-Tronic

\begin{tabular}{llll}
\hline Gear & Gear ratio & $\begin{array}{l}\text { Interval of } \\
\text { gear ratio }\end{array}$ & $\begin{array}{l}\text { Transmission } \\
\text { efficiency }\end{array}$ \\
\hline Reverse gear & -4.932 & & 0.9407 \\
1st gear & 5.503 & & 0.9596 \\
2nd gear & 3.333 & 1.65 & 0.9790 \\
3rd gear & 2.315 & 1.44 & 0.9698 \\
4th gear & 1.661 & 1.39 & 0.9797 \\
5th gear & 1.211 & 1.37 & 0.9877 \\
6th gear & 1 & 1.21 & 1 \\
7th gear & 0.865 & 1.16 & 0.9919 \\
8th gear & 0.717 & 1.21 & 0.9913 \\
9th gear & 0.601 & 1.19 & 0.9813
\end{tabular}

Table 13 The gear ratios, interval of gear ratio and transmission efficiency of GM 9T50E

\begin{tabular}{llll}
\hline Gear & Gear ratio & $\begin{array}{l}\text { Interval of gear } \\
\text { ratio }\end{array}$ & $\begin{array}{l}\text { Transmission } \\
\text { efficiency }\end{array}$ \\
\hline Reverse gear & -2.96 & & 0.9700 \\
1st gear & 4.69 & & 0.9702 \\
2nd gear & 3.31 & 1.42 & 0.9803 \\
3rd gear & 3.01 & 1.1 & 0.9665 \\
4th gear & 2.45 & 1.23 & 0.9703 \\
5th gear & 1.92 & 1.27 & 0.9766 \\
6th gear & 1.45 & 1.33 & 0.9907 \\
7th gear & 1 & 1.45 & 1 \\
8th gear & 0.75 & 1.34 & 0.9922 \\
9th gear & 0.62 & 1.21 & 0.9884 \\
\hline
\end{tabular}

method and the torque method, which are shown in Tables 11, 12 and 13.

Figure 18 presents the comparative analysis of ranges of gear ratios of the seven AT mechanisms. It is observed that the range of gear ratios of the $9 \mathrm{~T} 50 \mathrm{E}$ is the lowest, while that of the novel AT mechanism (3) is the highest. Moreover, it is found that there is no big difference in the range of gear ratios between the four novel ATs and the three existing ATs, which means that the ranges of gear ratios of the novel ATs meet the practical application requirements.

Figure 19 shows the comparative analysis of the intervals of gear ratios of the seven AT mechanisms. It is observed that the intervals of gear ratios of the novel ATs (3) and (4) fluctuate greatly, which means that the shift performance is poor. There are intervals of gear ratios bigger than 1.6 and smaller than 1.1 for novel ATs (2), (3), (4) and existing ATs of 9HP and 9G-Tronic. Therefore, the intervals of gear ratios of novel AT (1) and 9T50E are in completely consistent with the empirical value of 
1.1-1.6, which has a significant influence on the smooth shifting and comfortable driving.

Figure 20 shows the comparative analysis of transmission efficiencies of the seven AT mechanisms. The transmission efficiencies of all ATs satisfy the constraint conditions. Besides the reverse gear and 1st gear, the transmission efficiencies of other gears of the novel ATs are relatively high, which are not very different with the existing ATs.

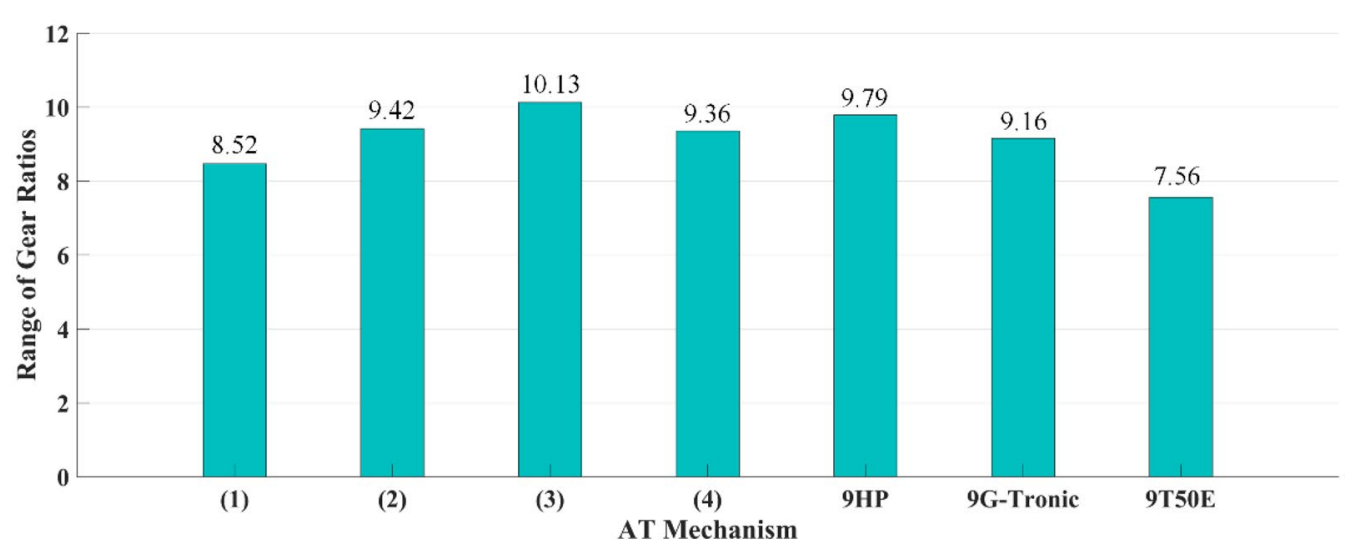

Figure 18 Comparative analysis of the ranges of gear ratios of the seven AT mechanisms

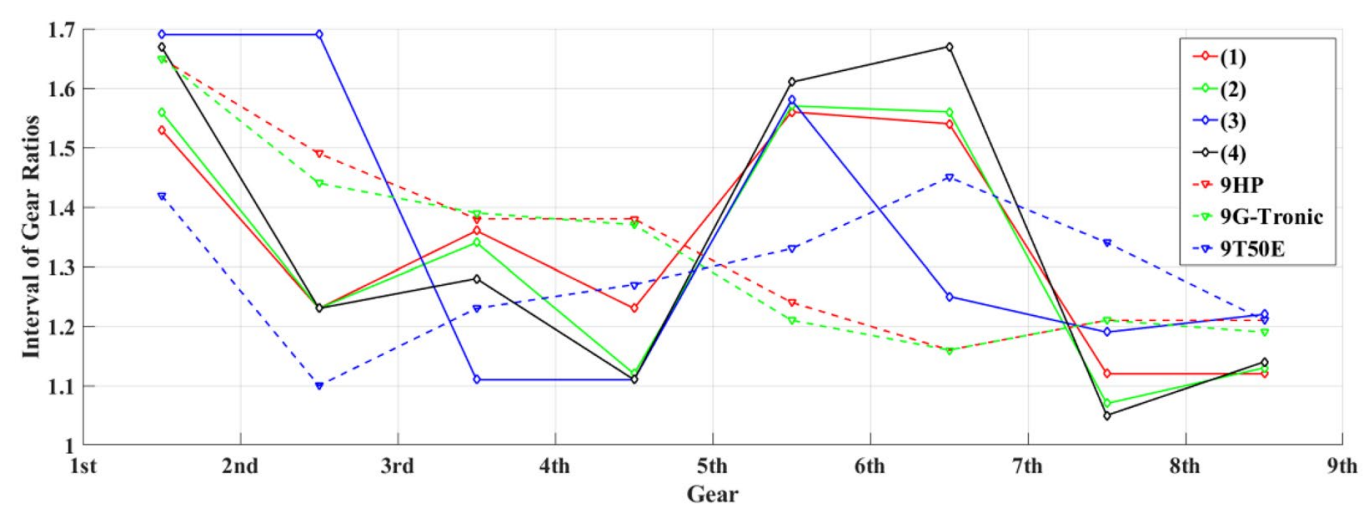

Figure 19 Comparative analysis of the intervals of gear ratios of the seven AT mechanisms

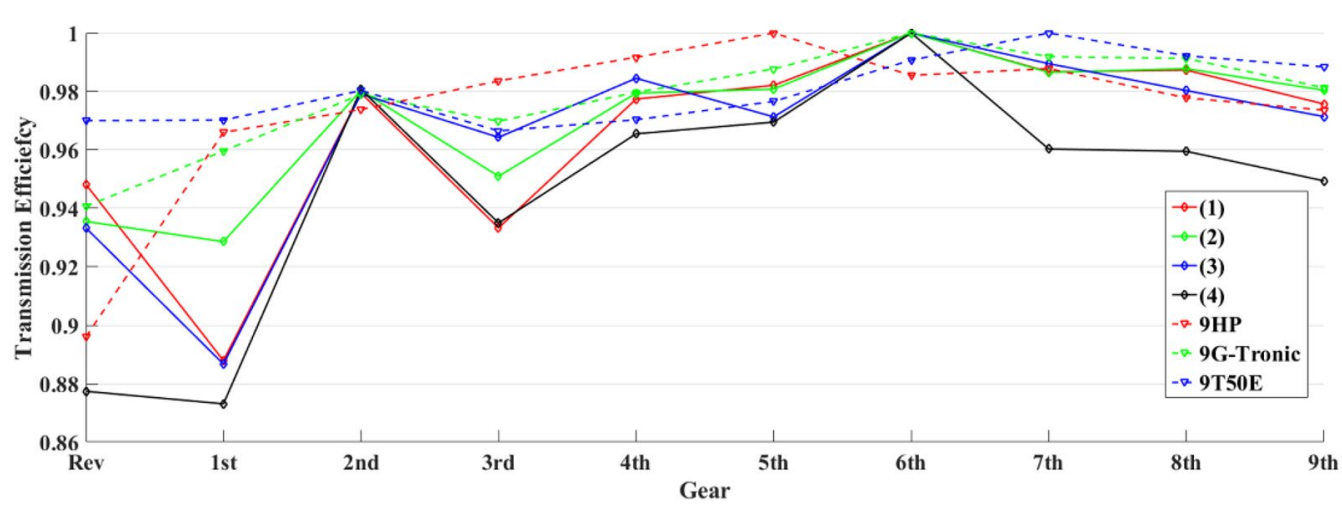

Figure $\mathbf{2 0}$ Comparative analysis of the transmission efficiencies of the seven AT mechanisms 
Table 14 Teeth number of gears in the PGSs

\begin{tabular}{lllll}
\hline & PGS $_{\mathbf{1}}$ & $\mathbf{P G S}_{\mathbf{2}}$ & $\mathbf{P G S}_{\mathbf{3}}$ & $\mathbf{P G S}_{\mathbf{4}}$ \\
\hline Sun gear & 50 & 24 & 50 & 30 \\
Planet gear & 10 & 24 & 10 & 18 \\
Ring gear & 70 & 72 & 70 & 66 \\
\hline
\end{tabular}

The comparative analysis of the range of gear ratios, interval of gear ratios and transmission efficiencies show that the four novel ATs are not very different from the existing ATs, which means that they are suitable for the practical application. It should be indicated that the transmission efficiency under the reverse gear and 1st gear will be optimized in the future work. During the novel ATs, only the novel AT mechanism (1) meets all of the constraint conditions completely. Therefore, the novel AT mechanism (1) is further analyzed and its prototype is manufactured for the speed test.

\section{Prototype Test}

Table 14 shows that the teeth numbers of gears are determined according to the definition of characteristic parameters of PGSs and the selected values described at Section 3.4.1. It should be indicated that standard spur gears are used. The modulus and the pressure angle of the gears are 2 and $20^{\circ}$, respectively.

Figure 21 shows the 3D model and the prototype of the AT mechanism. The structure of the prototype is simplified and the shifting elements are replaced with a simple device, which has the same function. For instance, the clutch plate has the same function as the clutch A when working with link $A_{1}$. Moreover, it has the same function as the clutch $B$ when working with link $B_{1}$. Furthermore, the component $C_{2}$ working with link $C_{1}$ has the same function as brake $C$, the component $D_{2}$ working with link $D_{1}$ has the same function as brake $D$, the component $E_{2}$ working with link $E_{1}$ has the same function as brake $E$ and the component $F_{2}$ working with link $F_{1}$ has the same function as brake F. It should be indicated that the function of shifting gears of the prototype is hand-actuated provisionally.

All of the shifting elements are separate at the beginning of the prototype test. The rotational speed of the input member is $18 \mathrm{r} / \mathrm{min}$. Table 5 shows the gear ranking. It is observed that the corresponding brakes and clutches are engaged and separated to achieve from the reverse gear to the ninth gear in turn. Figure 22 shows the working conditions of the prototype under each gear.

It should be indicated that the rotational speed of the output member under each gear is measured by a photoelectric velocimeter. Figure 23 shows that the measurement results are basically consistent with the theoretical

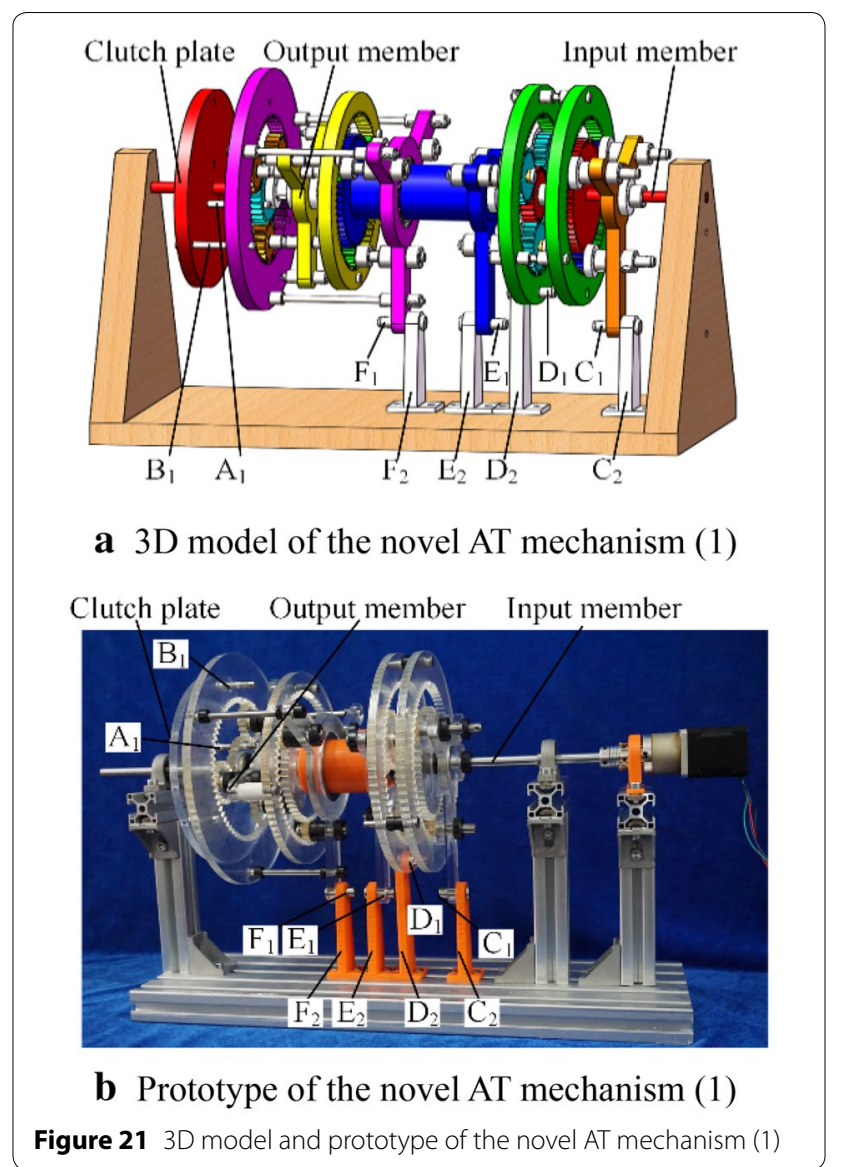

computing results. The obtained results prove the accuracy of the theoretical calculation and the feasibility of the AT mechanism.

\section{Conclusions}

In the present study, 9-speed ATs are synthesized based on the topological graph database of EGTs. Moreover, four novel AT mechanisms, which can achieve nine forward gears and one reverse gear are proposed. Their kinematics are analyzed theoretically based on the lever analogy method, and the gear ratios are obtained. The mechanical analysis is carried out according to the lever analogy method, which provides a theoretical basis for the fault diagnosis and development of the subsequent product. Furthermore, the power flow analysis is conducted based on the results of the kinematics and mechanical analysis. It should be indicated that the transmission efficiencies are calculated based on the torque method. The comparative analysis of the range of gear ratios, the interval of gear ratios and transmission efficiency between the four novel mechanisms and three existing ones are carried out. The obtained results show that the four novel mechanisms have the potential 


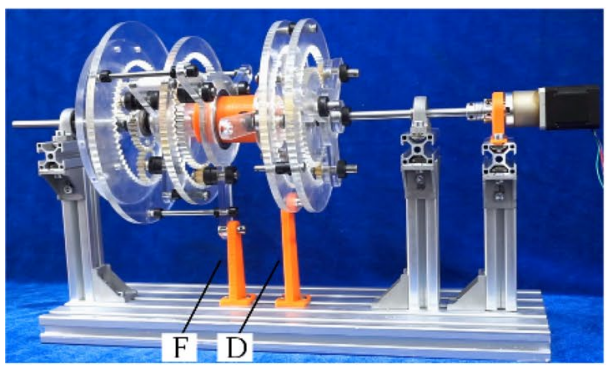

a The reverse gear

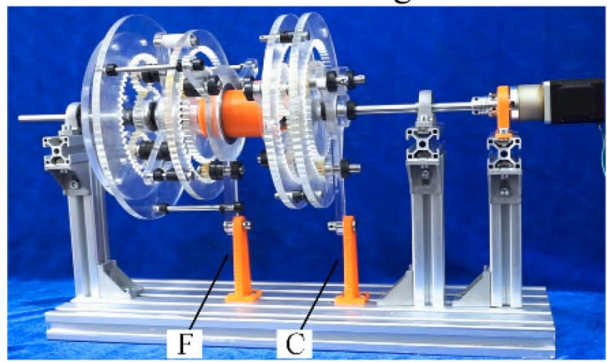

b The 1st gear

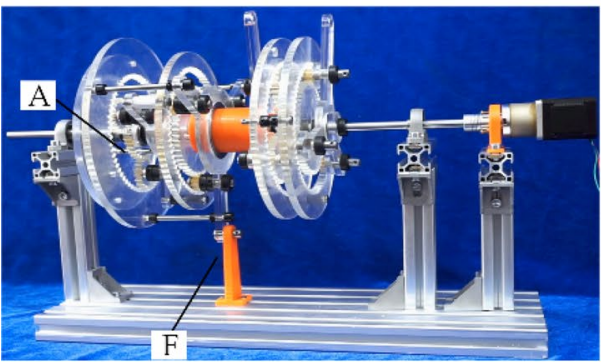

c The 2nd gear

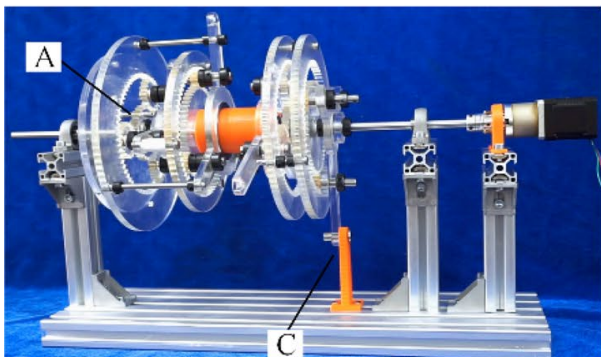

d The 3rd gear

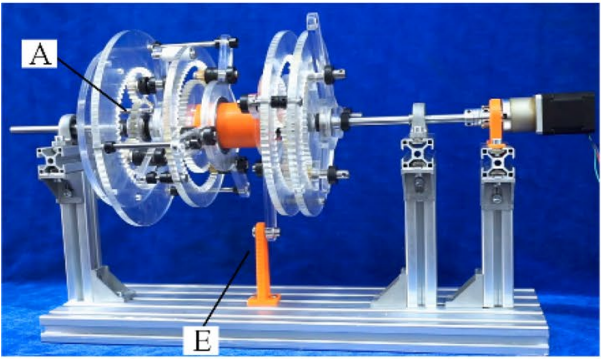

e The 4th gear

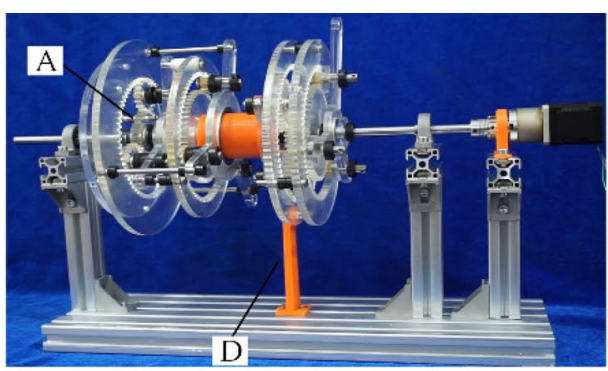

f The 5 th gear

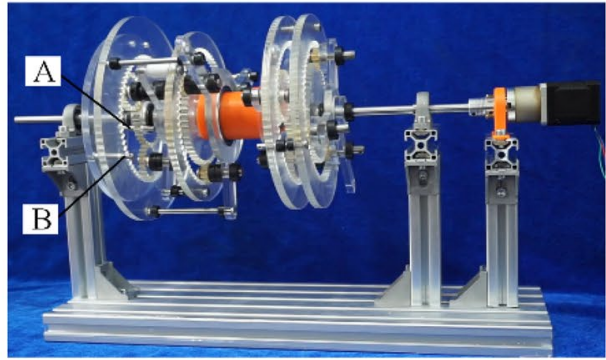

g The 6th gear

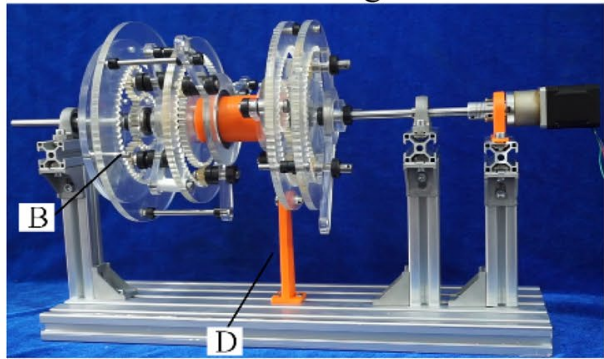

h The 7 th gear

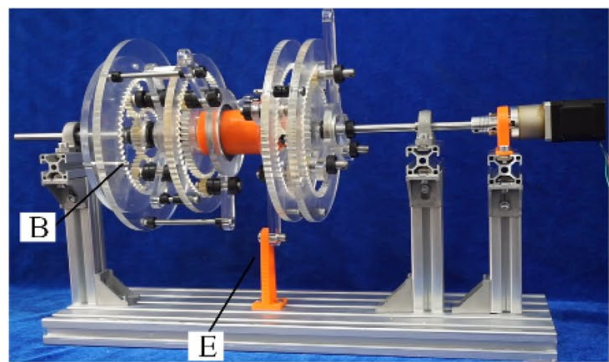

i The 8th gear

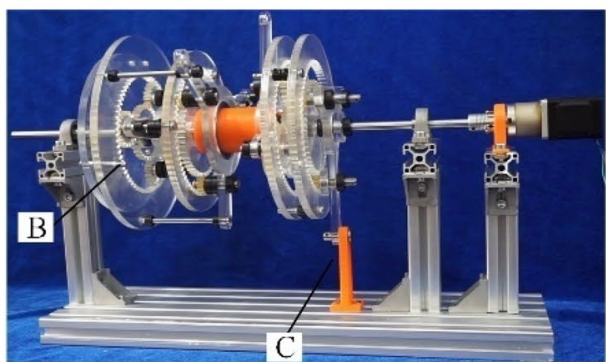

j The 9th gear

Figure 22 Working conditions of the prototype under each gear 


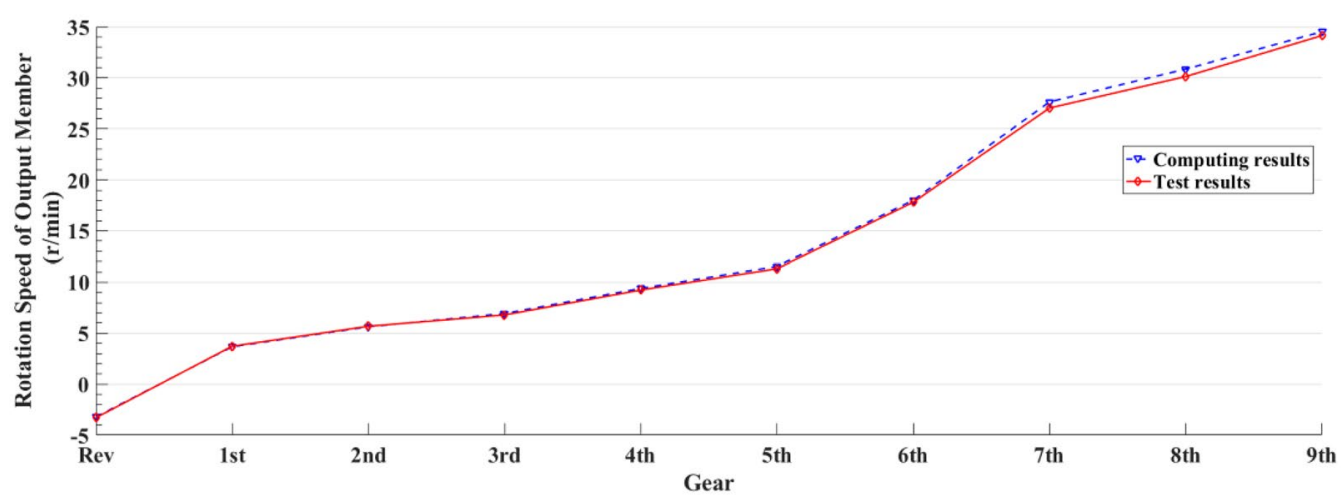

Figure 23 Comparative analysis of computing results and test results for the rotational speed of the output member

to be used in automatic transmissions. The novel AT mechanism with better performance is selected. The 3D model is established by taking a characteristic parameter set as example, and a prototype is manufactured. A speed test experiment is conducted. It is observed that the results are basically consistent with the theoretical computing results, which proves the accuracy of the theoretical calculation and the feasibility of the novel AT mechanisms. The dynamic analysis and optimal design for the prototype will be considered and conducted in the future work.

\section{Acknowledgements}

Not applicable.

\section{Authors' contributions}

$\mathrm{HD}$ and $\mathrm{ZC}$ were in charge of the whole trial; $\mathrm{CC}$ and TK wrote the manuscript; $\mathrm{CC}$ and $\mathrm{BM}$ manufactured the prototype and conducted the test. All authors read and approved the final manuscript.

\section{Authors' Information}

Huafeng Ding, born in 1977, is currently a professor at China University of Geosciences (Wuhan), China. He received his first PhD degree from Yanshan Universtiy, China, in 2007. He received the second PhD degree in the University of Duisburg-Essen, Germany, in 2015. His research interests include structural synthesis of mechanisms, conceptual design, and control and applications of planar and spatial mechanisms.

Changwang Cai, born in 1990, is currently a PhD candidate at the School of Mechanical Engineering, Yanshan University, China. He received his bachelor degree from Yanshan University, China, in 2013. His research interests include automatic transmission, configuration synthesis and mechanical engineering.

Ziming Chen, born in 1984, is currently an associate professor at the School of Mechanical Engineering, Yanshan University, China. He received his PhD degree from Yanshan University, China, in 2013. His research interests include design theory and application of parallel mechanisms.

Tao Ke, born in 1997, is currently a PhD candidate at school of mechanical and electronic information, China University of Geosciences (Wuhan), China. He received his bachelor degree from China University of Geosciences (Wuhan), China, in 2015. His research interests include mechanism design and analysis.

Bowen Mao, born in 1998, is currently an undergraduate at Yanshan University, China.
Funding

Supported by National Natural Science Foundation of China (Grant Nos. 51975544, 51675495).

\section{Competing interests}

The authors declare that they have no competing interests.

\section{Author Details}

${ }^{1}$ School of Mechanical Engineering, Yanshan University, Qinhuangdao 066004 China. ${ }^{2}$ Hebei Provincial Key Laboratory of Parallel Robot and Mechatronic System, Yanshan University, Qinhuangdao 066004, China. ${ }^{3}$ School of Mechanical Engineering and Electronic Information, China University of Geosciences (Wuhan), Wuhan 430074, China.

Received: 2 December 2019 Revised: 31 May 2020 Accepted: 11 June 2020

Published online: 26 June 2020

\section{References}

[1] W Gründler, H Mozer, F Sauter. Efficient torque converter automatic transmission for commercial vehicles. ATZ Worldwide, 2017, 119(6): 36-39.

[2] A Kahraman, H Ligata, K Kienzle, et al. A kinematics and power flow analysis methodology for automatic transmission planetary gear trains. Journal of Mechanical Design, 2004, 126(6): 1071-1081.

[3] R C Johnson, KTowfigh. Creative design of epicyclic gear trains using number synthesis. Journal of Engineering for Industry, 1967, 89(2): 309-314.

[4] F Buchsbaum, F Freudenstein. Synthesis of kinematic structure of geared kinematic chains and other mechanisms. Journal of Mechanisms, 1970, 5(3): 357-392.

[5] F Freudenstein. An application of Boolean algebra to the motion of epicyclic drives. Journal of Engineering for Industry, 1971, 93(1): 176-182.

[6] R Ravisankar, TS Mruthyunjaya. Computerized synthesis of the structure of geared kinematic chains. Mechanism and Machine Theory, 1985, 20(5): 367-387.

[7] LWTsai. An application of the linkage characteristic polynomial to the topological synthesis of epicyclic gear trains. Journal of Mechanisms, Transmissions, and Automation in Design, 1987, 109(3): 329-336.

[8] LWTsai, C C Lin. The creation of nonfractionated, two-degree-of-freedom epicyclic gear trains. Journal of Mechanisms, Transmissions, and Automation in Design, 1989, 111(4): 524-529.

[9] G Chatterjee, LWTsai. Enumeration of epicyclic-type automatic transmission gear trains. SAE Technical Paper 941012, 1994: 153-164.

[10] LW Tsai. Mechanism design: enumeration of kinematic structures according to function. CRC Press, London, 2000 
[11] J U Kim, B M Kwak. Application of edge permutation group to structural synthesis of epicyclic gear trains. Mechanism and Machine Theory, 1990, 25(5): 563-574.

[12] C H Hsu, KT Lam. A new graph representation for the automatic kinematic analysis of planetary spur-gear trains. Journal of Mechanical Design, 1992, 114(1): 196-200.

[13] C H Hsu, J J Hsu. An efficient methodology for the structural synthesis of geared kinematic chains. Mechanism and Machine Theory, 1997, 32(8): 957-973.

[14] J M del Castillo. Enumeration of 1-DOF planetary gear train graphs based on functional constraints. Journal of Mechanical Design, 2002, 124(4): 723-732.

[15] HT Ngo, H S Yan. Configuration synthesis of series-parallel hybrid transmissions. Proceedings of the Institution of Mechanical Engineers, Part D: Journal of Automobile Engineering, 2015, 230(5): 664-678.

[16] I Rajasri, A V S S K S Guptha, Y V D Rao. Generation of egts: hamming number approach. Procedia Engineering, 2016, 144: 537-542.

[17] V V Kamesh, K MallikarjunaRao, A B Srinivasa Rao. Topological synthesis of epicyclic gear trains using vertex incidence polynomial. Journal of Mechanical Design, 2017, 139(6): 062304.

[18] V R Shanmukhasundaram, Y V D Rao, S P Regalla. Enumeration of displacement graphs of epicyclic gear train from a given rotation graph using concept of building of kinematic units. Mechanism and Machine Theory, 2019, 134: 393-424.

[19] T Barhoumi, D Kum. Automatic enumeration of feasible kinematic diagrams for split hybrid configurations with a single planetary gear. Journal of Mechanical Design, 2017, 139(8): 083301.

[20] X Y Xu, H Q Sun, Y F Liu, et al. Automatic enumeration of feasible configuration for the dedicated hybrid transmission with multi-degree-offreedom and multiplanetary gear set. Journal of Mechanical Design, 2019, 141(9): 093301.

[21] TT Ho, S J Hwang. Configuration synthesis of two-mode hybrid transmission systems with nine-link mechanisms. Mechanism and Machine Theory, 2019, 142: 103615.

[22] Z Lévai. Structure and analysis of planetary gear trains. Journal of Mechanisms, 1968, 3(3): 131-148

[23] H L Benford, M B Leising. The lever analogy: a new tool in transmission analysis. SAE Technical Paper 810102, 1981:1-10.

[24] M F Gao, J B Hu. Kinematic analysis of planetary gear trains based on topology. Journal of Mechanical Design, 2018, 140(1): 012302.

[25] F C Yang, J X Feng, H C Zhang. Power flow and efficiency analysis of multi-flow planetary gear trains. Mechanism and Machine Theory, 2015, 92: 86-99.

[26] F Yang, J Feng, F Du. Design and power flow analysis for multi-speed automatic transmission with hybrid gear trains. International Journal of Automotive Technology, 2016, 17(4): 629-637.

[27] E L Esmail, E Pennestrì, A Hussein Juber. Power losses in two-degreesof-freedom planetary gear trains: a critical analysis of Radzimovsky's formulas. Mechanism and Machine Theory, 2018, 128: 191-204.

[28] Y H Cui, J Gao, X M Ji, et al. The multi-attribute topological graph method and its application on power flow analysis in closed planetary gear trains. Advances in Mechanical Engineering, 2018, 10(8): 1-9.

[29] K Arnaudov, D Karaivanov. The torque method used for studying coupled two-carrier planetary gear trains. Transactions of FAMENA, 2013, 37(1): 49-61.
[30] R A Lloyd. Power flow and ratio sensitivity in differential systems. Proceedings of the Institution of Mechanical Engineers, Part D: Journal of Automobile Engineering, 1991, 205(1): 59-67.

[31] J M del Castillo. The analytical expression of the efficiency of planetary gear trains. Mechanism and Machine Theory, 2002, 37(2): 197-214.

[32] H F Ding, J Zhao, Z Huang. Unified topological representation models of planar kinematic chains. Journal of Mechanical Design, 2009, 131(11): 114503.

[33] H F Ding, S Liu, P Huang, et al. Automatic structural synthesis of epicyclic gear trains with one main shaft. ASME 2015 International Design Engineering Technical Conference \& Computers and Information in Engineering Conference, Boston, Massachusetts, USA, 2-5 August 2015.

[34] W J Yang, H F Ding, B Zi, et al. New graph representation for planetary gear trains. Journal of Mechanical Design, 2018, 140(1): 012303.

[35] W J Yang, H F Ding. The perimeter loop-based method for the automatic isomorphism detection in planetary gear trains. Journal of Mechanical Design, 2018, 140(12): 123302

[36] W J Yang, H F Ding. The complete set of one-degree-of-freedom planetary gear trains with up to nine links. Journal of Mechanical Design, 2019, 141(4): 043301.

[37] J X Liu, L D Yu, Q L Zeng, et al. Synthesis of multi-row and multi-speed planetary gear mechanism for automatic transmission. Mechanism and Machine Theory, 2018, 128: 616-627.

[38] M F You, G Q Hou, M Y Wang. The analysis of the transmission scheme of the 9 speed automatic transmission based on the expend lever method. Advanced Materials Research, 2014, 945-949: 811-817.

[39] C Dörr, H Kalczynski, A Rink, et al. Nine-speed automatic transmission 9G-Tronic by Mercedes-Benz. ATZ Worldwide, 2014, 116(1): 20-25.

[40] T Martin, J Hendrickson. General Motors Hydra-Matic 9T50 automatic transaxle. SAE Technical Paper 2018-01-0391, 2018: 1-9.

[41] LW Tsai, E R Maki, T Liu, et al. The categorization of planetary gear trains for automatic transmissions according to kinematic topology. SAE Technical Paper Series 885062, 1988: 1513-1521.

[42] ZY Huang. Theory and design for modern AT. Shanghai: Tongji University Press, 2006. (in Chinese)

[43] P Johansen, D B Roemer, T O Andersen, et al. Morphological topology generation of a digital fluid power displacement unit using ChebychevGrübler-Kutzbach constraint. IEEE International Conference on Fluid Power and Mechatronics, Harbin, China, 5-7 August 2015: 227-230.

[44] H F Ding, C W Cai, W J Han, et al. Improved nine-gear transmission: CN, 201510424619.X. 2015-07-17.

[45] H F Ding, P Huang, C K Zhang, et al. Nine-gear speed changer: CN, 201510424662.6. 2015-07-17.

[46] H F Ding, CW Cai, H B Li. Nine-gear speed changer: CN, 201910138581.8. 2019-02-25.

[47] H F Ding, C W Cai, H B Li. A nine-gear speed changer: CN 201910137992.5. 2019-02-25.

[48] M Li, L Y Xie, HY Li, et al. Life distribution transformation model of planetary gear system. Chinese Journal of Mechanical Engineering, 2018, 31(1): 24-31.

[49] Y Q Wan, T L Liu. Planetary transmission scheme selection theory and optimization. Beijing: National Defense Industry Press, 1997. (in Chinese) 